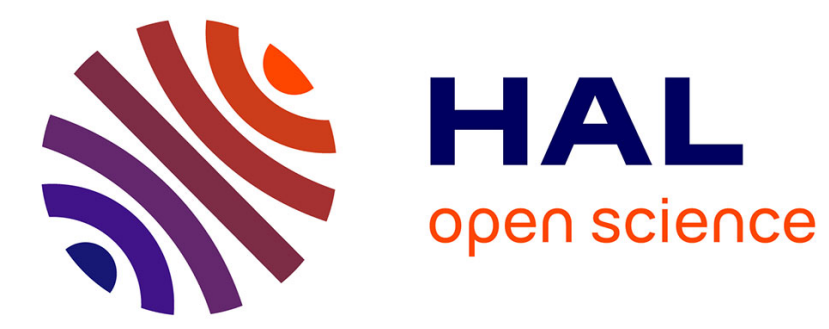

\title{
Médulla rénale
}

Lise Bankir, Nadine Bouby

\section{To cite this version:}

Lise Bankir, Nadine Bouby. Médulla rénale. Néphrologie \& Thérapeutique, 2016, 10.1016/j.nephro.2016.02.010 . hal-01290683

\section{HAL Id: hal-01290683 \\ https://hal.sorbonne-universite.fr/hal-01290683}

Submitted on 18 Mar 2016

HAL is a multi-disciplinary open access archive for the deposit and dissemination of scientific research documents, whether they are published or not. The documents may come from teaching and research institutions in France or abroad, or from public or private research centers.
L'archive ouverte pluridisciplinaire HAL, est destinée au dépôt et à la diffusion de documents scientifiques de niveau recherche, publiés ou non, émanant des établissements d'enseignement et de recherche français ou étrangers, des laboratoires publics ou privés. 


\title{
MÉDULLA RÉNALE
}

\section{RENAL MEDULLA}

\author{
Lise BANKIR et Nadine BOUBY \\ INSERM UMR-S 1138, Centre de Recherche des Cordeliers, \\ 75006 Paris, France; \\ Université Pierre et Marie Curie, 75006 Paris, France; \\ Université Paris-Descartes, 75006, Paris, France
}

Titre court : Médulla rénale

\section{Auteur correspondant}

Lise BANKIR, INSERM UMR-S 1138,

Centre de Recherche des Cordeliers

15 rue de l'Ecole de Médecine, 75006 PARIS, France

Tel : 33147353415 or 33682948010

E-mail : lise.bankir@inserm.fr 


\section{Résumé}

Le fait de pouvoir produire une urine hyperosmotique au plasma permet aux mammifères, y compris l'homme, d'excréter dans l'urine des déchets solubles, minéraux et organiques, en minimisant les besoins en eau. La capacité de concentration de l'urine dépend en premier lieu d'une disposition anatomique particulière en "épingle à cheveux" des néphrons et de certains vaisseaux, propre aux mammifères. Elle dépend aussi de l'action de l'hormone antidiurétique qui agit sur la perméabilité à l'eau du canal collecteur, sur toute sa longeur, et sur la perméabilité à l'urée dans sa partie tout à fait terminale, dans la médulla interne. Elle nécessite aussi: $1^{\circ}$ ) un "moteur" produisant un transport actif capable de générer une différence de concentration transépithéliale, créant une augmentation de la concentration de solutés dans l'interstitium environnant; et $2^{\circ}$ ) l'expression de transporteurs ou de canaux sélectifs localisés de façon très spécifique sur des portions limitées de certains segments du néphron, des canaux collecteurs et des vasa recta artériels, permettant d'accélérer considérablement le transport d'eau ou le passage de certains solutés à travers les membranes cellulaires.

Même si certains segments du néphron et du système collecteur sont présents dans le cortex et dans la médulla (notamment le tubule proximal, le segment large ascendant et le canal collecteur), leurs fonctions dans ces différentes zones peuvent ne sont pas totalement identiques en raison de leur environnement péritubulaire différent, de la composition du fluide qui les traverse, et de certaines différences de l'épithélium qui les borde. Cet article décrit quelques caractéristiques et fonctions particulières de ces structures dans la médulla rénale, par opposition aux structures correspondantes dans le cortex. Ces fonctions spécifiques des segments de néphron et du canal collecteur dans la médulla ont des conséquences qui peuvent se révéler néfastes dans certaines situations pathologiques.

\section{Mots-Clé}

Hormone anti-diurétique, urée, pars recta, segment large ascendant, canal collecteur, sécrétion, dilution, contre-courant 


\section{Abstract}

The ability to produce hyperosmotic urine allows mammals, including humans, to excrete their soluble mineral and organic waste products in the urine with a limited amount of water. The urinary concentrating capacity depends primarily on a special "loop-shaped" architecture of the nephrons and vessels, observed only in mammals. It also depends on the influence of antidiuretic hormone on the permeability to water of the collecting ducts on their entire length, and on the permeability to urea limited to the terminal portion of these ducts in the inner medulla. The ability to concentrate urine also requires (1) an "engine" able to produce an active (energy demanding) transport that generates a transepithelial concentration difference leading to increase the solute concentration in the surrounding interestitium; and (2) the expression of several membrane transporters or channels localized very specifically to limited portions of some nephron segments, collecting ducts and arterial vasa recta. These transporters and channels greatly accelerate the transport of water and some solutes accross the cell membranes.

Even if some nephron segments and parts of the collecting system are present in both the renal cortex and medulla (namely, the proximal tubule, the thick ascending limb and the collecting duct), their functions in the two renal zones may not be strictly similar, owing to their different peritubular environment, to the composition of the fluid running in their lumen, and to some differences in their epithelium. This paper describes some characteristics and specific functions of these structures in the renal medulla, as opposed to their corresponding structures in the cortex. These specific functions operating in the medulla may lead to various adverse consequences in some pathological situations.

\section{Key Words}

Antidiuretic hormone, urea, pars recta, thick ascending limb, collecting duct, secretion, dilution, counter-current 


\section{Abréviations}

Zones rénales et segments de néphron. Nomenclature en anglais selon les recommandations de l'International Union of Physiological Sciences $(1,2)$

$\begin{array}{lll}\text { Abrév. } & \text { Termes en francais } & \text { Termes en anglais } \\ \text { C } & \text { Cortex } & \text { Cortex } \\ \text { OM } & \text { Médulla externe } & \text { Outer medulla } \\ \text { OS } & \text { Zone externe de la OM } & \text { Outer stripe of OM } \\ \text { IS } & \text { Zone interne de la OM } & \text { Inner stripe of OM } \\ \text { IM } & \text { Médulla interne } & \text { Inner medulla } \\ \text { MR } & \text { Rayon médullaire } & \text { Medullary ray } \\ \text { PCT } & \text { Tubule contourné proximal } & \text { Proximal convoluted tubule } \\ \text { PST } & \text { Pars recta (du tub. proximal) } & \text { Proximal straight tubule } \\ \text { DTL } & \text { Segment grèle descendant } & \text { Descending thin limb } \\ \text { ATL } & \text { Segment grèle ascendant } & \text { Ascending thin limb } \\ \text { TAL } & \text { Segment large ascendant } & \text { Thick ascending limb } \\ \text { MTAL } & \text { TAL médullaire } & \text { Medullary TAL } \\ \text { CTAL } & \text { TAL cortical } & \text { Cortical TAL } \\ \text { DCT } & \text { Tubule contourné distal } & \text { Distal convoluted tubule } \\ \text { CD } & \text { Canal collecteur } & \text { Collecting duct } \\ \text { CCD } & \text { CD cortical } & \text { Cortical CD } \\ \text { OMCD } & \text { CD de la OM } & \text { Outer medullary CD } \\ \text { IMCD } & \text { CD de la IM } & \text { Inner medullary CD } \\ \text { VR } & \text { Vasa recta } & \text { Vasa recta } \\ \text { MIC } & \text { Cellules interstitielles de la IM } & \text { Interstitial medullary cells }\end{array}$

\section{Autres abréviations}

$\begin{array}{ll}\text { AQP } & \text { Aquaporine } \\ \text { ADH } & \text { Hormone anti-diurétique } \\ \text { AVP } & \text { Vasopressine } \\ \text { dDAVP } & \text { dé-amino 8-D arginine vasopressine (agoniste des récepteurs V2) } \\ \text { DFG } & \text { Débit de filtration glomérulaire } \\ \text { ENaC } & \text { Canal sodium épithélial } \\ \text { NKCC2 } & \text { Co-transporteur } \mathrm{Na} / \mathrm{K} / 2 \mathrm{Cl} \\ \text { UT } & \text { Transporteur facilité d'urée }\end{array}$




\section{Introduction}

Le fait de pouvoir produire une urine hyperosmotique au plasma permet aux mammifères, y compris l'homme, d'excréter dans l'urine des déchets solubles, minéraux et organiques, en minimisant les besoins en eau. Si cette fonction de concentration a eu des avantages évidents au cours de l'évolution, elle est par ailleurs impliquée dans certaines pathologies, soit directement (risque de lithiase rénale), soit indirectement, en contribuant à la progression de l'insuffisance rénale, à la néphropathie diabétique, à la polykystose rénale autosomique dominante, et elle est associée à un risque accru de maladies métaboliques et cardiovasculaires (3).

La capacité de concentration de l'urine dépend en premier lieu d'une disposition anatomique particulière des néphrons propre aux mammifères. Tous les vertébrés possèdent une hormone antidiurétique (ADH) très bien conservée au cours de l'évolution (nonapeptide avec une structure cyclique due à un pont di-sulfure). Chez tous les vertébrés aussi, du poisson-zèbre à l'homme, les segments successifs qui forment les néphrons et le système collecteur ont à peu près les mêmes propriétés et sont équipés de transporteurs membranaires ayant des fonctions équivalentes ou très proches. C'est ce qui a permis par exemple d'en cloner certains (par la technique d'expression fonctionnelle) à partir du rein d'amphibien avant de cloner ensuite, par homologie, leurs équivalents murins et humain. Mais seuls les mammifères ont un rein dans lequel une distinction très nette existe entre "cortex" et "médulla". Cette distinction est due au repliement des néphrons en forme d'épingle à cheveux formant les "anses de Henle" (du nom de l'anatomiste allemand qui les a décrites en premier). Dans ces anses, le fluide tubulaire circule en parallèle et à contre-courant dans une branche "descendante" et une branche "ascendante". Les vaisseaux irriguant et drainant la médulla sont également orientés de la même façon. C'est cette disposition anatomique particulière qui permet aux mammifères de produire une urine hyperosmotique au plasma et ainsi d'excréter les déchets solubles dans l'urine en minimisant les besoins en eau.

Nous avons choisi d'utiliser dans cet article les abréviations internationales correspondants aux différentes zones rénales et aux différents segments du néphron, en suivant les recommandations de nomenclature proposées par l' "Union of Physiological Sciences" et publiées dans plusieurs journaux simultanément, sans copyright $(1,2)$, puis reprises ensuite dans plusieurs ouvrages. 
Remarque 1. L'hormone antidiurétique s'appelle aussi vasopressine car les premiers travaux sur des extraits hypophysaires, en France d'une part, et aux Etats Unis d'autre part, avaient mis en évidence deux actions bien distinctes avant qu'on découvre qu'il s'agissait de la même hormone agissant sur des récepteurs différents (récepteurs V2 pour les effets antidiurétiques et V1a pour les effets vasoconstricteurs). Dans cet article, nous parlerons préférentiellement de l'ADH quand il s'agira de la fonction de concentration de l'urine, mais parfois d'AVP ou de vasopressine quand des effets médiés par les récepteurs V1a seront évoqués.

Remarque 2. L'abréviation acceptée internationalement pour l'hormone antidiurétique ou vasopressine est "arginine-vasopressin" ou AVP. Le "A" a été conservé même si le mot "arginine" (acide aminé en position 8) n'a plus de raison d'être. Avant qu'on dispose d'hormone synthétique, on utilisait à des fins expérimentales un extrait d'hypophyse de porc. Le "A" permettait de distinguer la forme la plus courante de vasopressine chez presque tous les mammifères de la "lysine-vasopressine" (LVP) qui n'existe que chez les suidés (porc, sanglier, phacochère). On utilise maintenant de la vasopressine de synthèse ou des analogues peptidiques divers, synthétiques eux aussi, mais toujours avec l'arginine en position 8 et non de la lysine. La substitution d'une lysine à la place de l'arginine ne modifie pas l'affinité de l'hormone pour ses récepteurs ni la réponse fonctionnelle des tissus-cible.

Remarque 3. "Anse de Henle" se prononce "henlé" mais s'écrit sans accent car le "e" en allemand se prononce "é".

\section{La médulla: ses différentes zones et leur vascularisation spécifique}

L'organisation très particulière de la médulla rénale des mammifères, ses conséquences fonctionnelles, et les différences entre espèces ont été très bien étudiées et décrites par Wilhelm Kriz, Brigitte Kaissling et coll. dans de nombreux articles, revues et chapîtres (4-7).

\section{Le cortex et les trois zones de la médulla}

Par définition, les glomérules de tous les néphrons sont situés dans la partie la plus externe du rein, le cortex (voir Figure 1). La médulla contient uniquement des 
anses de Henle, des vasa recta (peu de capillaires proprement dits), des canaux collecteurs et des cellules interstitielles. Les mécanismes qui poussent les ébauches de néphrons à former des "anses" au cours de la formation du métanéphros pendant l'embryogénèse ne sont pas bien compris (8).

Dans un rein de mammifère typique, et entre autres chez les rongeurs (dont les rats et souris utilisés en laboratoires), la médulla comporte trois zones, bien visibles sur les Figures 1a et 1b. Leurs limites sont définies par les transitions entre différents segments du néphron. Comme l'illustre la Figure 2, la zone externe de la médulla externe (outer stripe = OS) contient les "pars recta" (PST), partie terminale des tubules proximaux, assez rectiligne pour les néphrons superficiels et plus tortueuse (ce qui augmente leur longeur) pour les néphrons juxtamédullaires. La transition, toujours très brusque, entre les PST et les segments grèles descendants (TDL) définit la limite entre les zones externe et interne de la médulla externe. La zone interne de la médulla externe (inner stripe = IS) contient les segments grèles descendants et les segments larges ascendants (TAL) de tous les néphrons, tandis que la médulla interne (inner medulla $=\mathrm{IM}$ ) ne contient que des segments grèles descendants et ascendants (ATL) appartenant aux néphrons dits "à anse longue", et dont les glomérules sont généralement situés dans le cortex profond (9). On appelle "papille" la partie profonde de la médulla interne (IM), en forme de cône.

Les canaux collecteurs (CD) traversent toutes les zones de la médulla pour s'aboucher à la pointe de papille et déverser l'urine dans le bassinet. Notez, comme le montre la Figure 2, que ces canaux traversent la médulla externe sans converger entre eux. C'est seulement dans la médulla interne qu'ils convergent en canaux de plus gros diamètre et moins nombreux.

La médulla du rein humain est multilobée, et chaque lobe (ou pyramide) correspond à la médulla d'un mammifère typique. Ce type de rein multipyramidal est peu courant chez les mammifères terrestres (10). Les pyramides de médulla interne sont assez courtes tandis que le cortex est assez épais.

On sait depuis longtemps que la hauteur de la médulla par rapport à celle du cortex est, en général, associée au pouvoir de concentration de l'urine. La médulla interne est proportionnellement très développée chez les rongeurs adaptés à la vie désertique, tandis que le castor, qui vit dans des rivières, a une médulla interne presque inexistante $(11,12)$. L'homme et les primates présentent une situation intermédiaire. Chez la plupart des mammifères il y a environ 70-85 \% de néphrons "à 
anse courte" (c'est à dire dont la pointe de l'anse est à la limite entre médulla externe et médulla interne) et 15-30\% de néphrons "à anse longue" dont l'anse pénètre à des profondeurs diverses dans la médulla interne. Mais certaines espèces dont la capacité à concentrer l'urine est très limitée ou relativement modeste, ont, parmi les néphrons à anse courte, une forte proportion de néphrons dits "corticaux", c'est à dire dont l'anse ne penêtre pas du tout dans la médulla. La pointe de l'anse tourne dans le cortex et il n'y a donc pas ou pratiquement pas de segment grèle descendant. Il est impropre d'appeler "néphrons corticaux" tous les néphrons dont le glomérule est situé dans le cortex superficiel. Les néphrons "corticaux" sont uniquement ceux dont l'anse tourne dans le cortex.

Des travaux de micro-dissection ou de microinjection de néphrons ont montré que, chez les rongeurs, les anses de Henle de tous les néphrons à anse courte pénètrent dans la médulla externe et tournent au même niveau. Par contre, chez I'homme, il y a une forte proportion de néphrons corticaux $(13,14)$ (Figure 3). Ils peuvent donc participer à la dilution de l'urine, mais pas à sa concentration (voir plus loin). La Figure 4 montre la longeur des anses de Henle de 70 néphrons dont on a pu suivre le parcours en remplissant leur lumière avec du silastic (Microfil silicone rubber()) par une micropipette insérée dans l'espace urinaire du glomérule. Environ $20 \%$ des néphrons (15/70) ne pénêtrent pas du tout ou à peine dans la médulla (néphrons corticaux), et $27 \%$ pénêtrent dans la médulla interne (néphrons à anse longue). Les $43 \%$ intermédiaires ont une anse qui tourne dans la médulla externe (néphrons à anse courte).

Dans le cortex, les parties "droites" des tubules proximaux et distaux des néphrons superficiels et moyens sont rassemblées par petits groupes autour de quelques canaux collecteurs (4 en général chez le rat), formant ainsi des "rayons médullaires" dans le cortex (voir Figure 1B). Ces rayons médullaires contrastent avec les zones qui les entourent latéralement, zones dites "labyrinthiques", dans lesquelles se trouvent les artéres et les veines interlobulaires, les glomérules et les tubules contournés proximaux et distaux. Les rayons médullaires ont la même composition tubulaire que la OS, et même en partie le même environnement vasculaire car les vasa recta veineux qui remontent de la médulla peuvent parfois, au lieu de rejoindre les veines arquées (à la jonction cortico-médullaire), pénétrer dans les rayons médullaires du cortex profond et se jeter dans les veines interlobulaires (comme on le voit de façon beaucoup plus marquée chez certains rongeurs adaptés à la vie désertique (15). 
Lors d'adaptations fonctionnelles associées à des modifications de régime alimentaire (apport en protéines, ou en potassium par ex), on a observé des modifications importantes de la hauteur relative (ou du volume relatif) des différentes zones rénales, généralement associées à une hypertrophie du rein $(16,17)$. Par exemple, lors d'une stimulation de l'activité de concentration de l'urine par la perfusion d'un agoniste de l'ADH, comme en réponse à un régime riche en protéines, la IS s'allonge et avec elle, tous les segments de néphron qu'elle contient. Ceci augmente la longeur du système de multiplication par contre-courant $(18,19)$. Tandis qu'au cours de l'hypertrophie compensatrice (après ablation totale d'un rein), l'hypertrophie du rein restant affecte toutes les zones rénales de façon homogène (20).

\section{Vascularisation de la médulla}

Toute la circulation médullaire dérive des artérioles efférentes des glomérules profonds (juxta-médullaires). Les anses de Henle ne sont pas irriguées par des vasa recta issus de leur propre artérielle efférente glomérulaire, comme il est trop souvent représenté dans les schémas. Elles sont irriguées par les vaisseaux efferents des glomérules de plusieurs néphrons moyens et profonds comme l'illustre très clairement le film "The Human Kidney" réalisé par Reiner Beeuwkes en 1975 (voir lien internet vers ce film à la fin de l'article). D'autre part, la vascularisation de la médulla n'est pas constituée seulement de vasa recta descendants (artériels) et montants (veineux). La zone interne de la médulla externe (IS) comporte des régions riches en capillaires (essentiels pour l'irrigation des segments larges ascendants médullaires) qui alternent avec les faisceaux de vasa recta (voir Figure 5). Donc, en plus de l'hétérogénéité axiale présente dans la médulla (le long de l'axe des néphrons), il existe une forte hétérogénéité transversale (juxtaposition latérale dans une même zone de la médulla). Les relations vasculo-tubulaires sont très différentes d'une zone à l'autre $(4-6,21,22)$, ce qui rend très difficile la modélisation mathématique de la fonction de concentration.

Il est courant de dire que la médulla a un débit sanguin très faible. En fait, le rein reçoit un pourcentage très élevé du débit cardiaque $(20 \%$, alors qu'il représente moins de $2 \%$ du poids corporel). Même s'il est proportionnellement faible par rapport au débit du cortex, le débit sanguin médullaire est, en moyenne, comparable à celui d'autres organes comme le muscle au repos (mais décroissant graduellement vers la profondeur). Mais sa teneur en oxygène s'appauvrit fortement en descendant dans la 
médulla car l'oxygène est shunté du fait des échanges par contre-courant qui se produisent entre vasa recta descendants et ascendants dans les faisceaux vasculaires (voir plus loin). Les TAL dans leur partie initiale, dans la IS, sont irrigués par un réseau de capillaires qui ne subissent pas ou peu ce shunt car les artérioles qui alimentent les plexus capillaires péritubulaires dans la IS sont à la périphérie des faiseaux vasculaires et s'en écartent rapidement. Par contre, les pars recta dans la OS reçoivent peu de sang artériel car les artérioles efférentes des glomérules profonds traversent la OS en formant peu de capillaires. Les pars recta sont principalement irriguées par les vasa recta veineux remontant de zones plus profondes de la médulla (Figure 6).

Dans la médulla interne, il existe, entre les anses de Henle, les canaux collecteurs et les vasa recta, un abondant tissu interestitiel qui forme un milieu environnant commun entre toutes ces structures. Comme les vasa recta veineux ont un endothélium fenestré, tous les solutés et l'eau peuvent, à chaque niveau traversé par le sang dans l'interstitium, s'équilibrer avec les concentrations présentes dans ces vasa recta. L'albumine peut aussi s'extravaser dans cet interstitium. D'autre part, les caractéristiques anatomiques de ce tissu interstitiel permettent de limiter la diffusion des solutés dans le sens axial, ce qui limite la dissipation des gradients osmotiques. Les cellules interstitielles possèdent des récepteurs à diverses hormones et médiateurs et relarguent elles mêmes des médiateurs qui agissent sur le débit sanguin de la médulla (voir plus loin).

\section{Conséquences fonctionnelles de la vascularisation de la OS}

Les vasa recta veineux (ascendants) qui irriguent les pars recta dans la OS sont largement fenestrés, et une partie d'entre eux a subi des échanges par contrecourant avec les vasa recta artériels dans les faisceaux vasculaires. Les autres remontent des plexus péritubulaires de la IS et sont appauvris en oxygène du fait de la consommation élevée des MTAL. Ceci explique pourquoi la OS est la zone qui souffre le plus d'anoxie en cas d'hypo-perfusion rénale. Il est intéressant de noter que c'est dans le cortex profond et la OS que sont situées de façon majoritaire les cellules interstitielles produisant l'érythropoiétine.

Il faut noter aussi que la OS est la zone où le risque de déficit en arginine (précurseur du NO) est le plus important (23). L'arginine d'origine alimentaire est complètement dégradée par le foie et c'est le tubule contourné proximal qui, à partir de la citrulline filtrée, produit la plus grande partie de l'arginine circulante $(24,25)$. 
Cette arginine néoformée est rapidement entraînée dans la circulation générale par l'abondant débit sanguin du cortex et n'est pas directement utilisée par le rein. Par contre, de l'arginine est consommée dans les pars recta pour produire de l'ornithine qui sert de substrat pour la synthèse de polyamines $(26,27)$. Le métabolisme des polyamines est particulièrement élevé dans la OS. Ces molécules protectrices sont importantes pour la fidélité de réplication de l'ADN, la formation d'ARN, et le maintien de l'intégrité cellulaire. L'intensité du métabolisme des polyamines dans le PST est très probablement importante pour assurer le mantien de l'intégrité cellulaire dans ce segment situé dans un environnement hostile (hyper-osmolarité, faible oxygénation, forte concentration en $\mathrm{NH}_{4}$, acide urique et autres acides organiques, etc.....) (27, 28).

La pars recta est un segment qui peut aussi souffrir d'un manque d'ATP en cas de consommation alimentaire importante de fructose (abondant dans les boissons sucrées). Car le fructose stimule des réactions biochimiques intracellulaires catalysées par la fructokinase, qui consomment beaucoup d'ATP (29).

\section{Contrôle multi-hormonal des fonctions tubulaires dans la médulla}

De très nombreuses hormones et médiateurs régulent les fonctions de transport des tubules situés dans la médulla et influencent leur irrigation vasculaire. Nous ne traiterons pas ici de ces régulations mais souhaitons seulement illustrer à quel point les sites d'action de certaines hormones sont très précisemment localisés dans les différentes zones de la médulla (et vraisemblablement dans des structures spécifiques à l'intérieur de ces zones). La Figure 7 montre (en fausses couleurs) la localisation des récepteurs de quatre hormones peptidiques, mise en évidence par autoradiographie après application, sur des coupes de rein de rat, d'hormones radiomarquées à l'iode ${ }^{125}$. La dDAVP, un agoniste sélectif des récepteurs V2 de la vasopressine, se fixe principalement sur les canaux collecteurs présents dans les trois zones de la médulla, avec une intensité plus forte dans la médulla interne. L'angiotensine II (All), le peptide natriurétique atrial (ANP) et l'endothéline (ET) se fixent sur les glomérules. L'All se fixe aussi de façon marquée dans la IS. II s'agit des faisceaux vasculaires, mais également, et moins fortement, de structures tubulaires dans cette même zone (probablement les TAL) (30). Par contre, elle ne se fixe pas du tout dans la OS et à peine dans la partie superficielle de la IM. L'ANP, elle, se fixe intensément dans la partie profonde de la IM (sans doute sur les canaux collecteurs terminaux) avec une quasi-absence de marquage dans les deux sous-zones de la 
médulla externe (31). Comme on le voit sur ces photos, ces trois hormones ne se fixent pas sur les structures présentes dans la OS. Par contre, les récepteurs de la PTH et ceux de la dopamine (pas de photo) sont sans doute abondants sur les pars recta dans la OS (d'après les résultats de travaux fonctionnels). Enfin, l'ET se fixe très fortement dans la IS et dans la IM, mais sans marquage dans la OS (32).

\section{Système de dilution et de concentration de l'urine. Les quatre étapes}

Remarque 4. II faut distinguer osmolaLité (concentration d'osmoles par kg d'eau) et osmolaRité (concentration d'osmoles par litre d'eau). Un litre d'eau dans laquelle sont dissous des solutés pèse plus qu'un $\mathrm{kg}$ (la densité de la solution est supérieure à 1). Mais en fait, dans la gamme des valeurs biologiques, la différence entre osmolarité et osmolalité n'est que de quelques pourcents. D'autre part, les cellules "voient" plutôt des concentrations d'osmoles par litre de fluide que par kilogramme de fluide. Aussi, sauf quand il s'agit de valeurs mesurées avec des osmomètres modernes qui donnent des résultats en mosm/kg H2O, on privilégiera le mot "osmolarité" dans cet article.

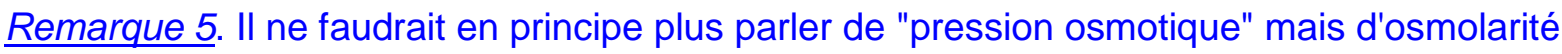
(ou osmolalité) car la concentration des solutés dissous dans un liquide n'a pas la dimension d'une pression. On employait ce terme quand on évaluait indirectement cette concentration par la différence de niveau de liquide entre deux récipients communiquant par une membrane semi-permeable, dont l'un était rempli d'eau pure et l'autre d'une solution inconnue. La différence de hauteur s'exprimait en termes de pression comme la pression atmosphérique.

On l'oublie souvent, mais avant de se concentrer dans la médulla, l'urine primitive subit au préalable une forte dilution qui est obligatoire. Cette dilution se produit même en absence totale de stimulation hormonale (comme observé en perfusion de tubule isolé ou par microponction du tubule distal précoce chez le rat "anhormonal" (c'est à dire un modèle dans lequel les hormones qui stimulent le TAL sont absentes ou neutralisées) $(33,34)$. Ensuite, si l'ADH est présente, l'urine sera "ré-équilibrée" dans le CD cortical jusqu'à égaler l'osmolarité du milieu intérieur. C'est en traversant ensuite les différentes zones de la médulla (en progressant dans la lumière des $\mathrm{CD}$ ) que l'urine pourra se concentrer au delà de ce niveau, à condition qu'une accumulation progressive de solutés ait pu créer une hyperosmolarité 
suffisante dans l'interstitium de la médulla. Cette accumulation dépend d'un mécanisme complexe, encore incomplètement élucidé, qui peut se décomposer en deux étapes. Ces 4 étapes (dilution, ré-équilibration, concentration dans la OM, puis dans la IM) vont être détaillées ci-dessous.

\section{Phase 1: Dilution}

La capacité de dilution existe chez tous les vertébrés. Les néphrons des amphibiens ne sont pas repliés en forme d'épingle à cheveux comme ceux des mammifères et n'ont pratiquement pas de segment grèle, mais ils ont, avant le tubule distal, un "segment de dilution" qui a beaucoup de caractéristiques morphologiques et de propriétés de transport identiques à celles du TAL des mammifères. Les amphibiens, qui pour beaucoup vivent en eau douce, peuvent diluer l'urine pour excreter de l'eau "libre" (c'est à dire de l'eau libre d'osmoles), et éventuellement selon les conditions, la re-concentrer ensuite jusqu'à l'iso-osmolarité en aval, sous l'action de la vasotocine (Figure 8). Chez l'homme, les néphrons "corticaux" qui ne pénètrent pas dans la médulla ont un TAL cortical, mais pas de TAL médullaire (Figures 3 et 4 ). Ils peuvent donc participer significativement à la dilution de l'urine, mais pas à la création d'un gradient osmotique dans la médulla. A l'inverse, les néphrons à anse longue (chez l'homme et tous les mammifères) participent à la création et au mantien de ce gradient osmotique dans la médulla, mais ne participent pas ou très peu à la dilution, car ils n'ont pratiquement pas de TAL cortical.

La capacité de dilution est conservée chez les mammifères quand la concentration d'ADH est nulle ou faible. Elle permet d'excréter de l'eau "libre" en plus ou moins grande quantité pour faire face à une ingestion importante de liquide (35, 36). C'est à cause de cette dilution obligatoire que, dans différentes formes de diabète insipide, l'absence d'ADH ou des mutations inactivant les récepteurs V2 ou I'AQP2 conduisent à des volumes considérables d'urines très largement hypoosmotiques (volumes urinaires quotidiens environ dix fois supérieurs à la normale). La simple absence de capacité de concentration, produisant une osmolarité urinaire proche de celle du plasma, conduirait seulement à doubler ou tripler le débit urinaire par rapport à des urines à 600 ou 900 mosm/L.

La fonction de dilution est principalement due à la réabsorption de $\mathrm{NaCl}$ par le NKCC2 dans le TAL (un segment imperméable à l'eau) et dans le CC cortical (par I'ENaC) (qui est impermeable à l'eau quand l'ADH est absente ou ne peut agir sur I'AQP2). Contrairement à ce qu'on pourrait penser, les hormones qui agissent en 
augmentant l'activité de ces transporteurs ne permettent pas de modifier la concentration de l'urine à la fin du TAL ou à la fin du CD cortical. Elles permettent seulement d'atteindre un point d'équilibre plus tôt le long de ces segments. Ils présentent en quelque sorte une "réserve fonctionnelle" sur une partie de leur longeur. Pour le TAL, ce point d'équilibre, c'est le "static head" défini par Maurice Burg (37), c'est à dire le point où se produit la différence maximum de concentration transépithéliale que le TAL peut produire sans laisser fuir quand même un peu d'eau hors de la lumière (malgré sa très faible permeabilité à l'eau) (37-39). Pour le CD cortical, l'équilibre se produit quand le fluide tubulaire atteint une osmolarité égale à celle du tissu interstitiel du cortex environnant (40-42).

\section{Phase 2: Ré-équilibration jusqu'à l'isoosmolarité}

Il est intéressant de souligner que la fonction de dilution joue aussi un rôle dans la concentration ultérieure de l'urine. Car, en présence d'ADH, l'urine se re-équilibre jusqu'à l'iso-osmolarité dans le CD cortical, permettant ainsi qu'une réabsorption d'eau importante se produise déjà dans le cortex (Figure 8). Ceci réduit fortement le débit du fluide tubulaire dans les CD corticaux et évite de faire entrer un excès d'eau lorsqu'ils pénètrent dans la médulla (43). Si l'osmolarité à la sortie de l'anse de Henle est de $145 \mathrm{mosm} / \mathrm{L}$ et celle de l'interstitium cortical de $290 \mathrm{mosm} / \mathrm{L}$, la réabsorption d'eau dans le CCD réduira le débit de moitié. De plus, l'ADH, par ses effets sur les récepteurs $\mathrm{V} 2$ du $C D$, stimule l'ENaC qui fait réabsorber du sodium, entraînant ainsi à sa suite un mouvement d'eau supplémentaire (40). Le processus de concentration qui a lieu dans la médulla sera plus efficace s'il s'exerce sur un volume de fluide plus réduit.

\section{Phase 3: Multiplication par contre courant dans la médulla externe}

La multiplication par contre-courant dans les anses de Henle (à ne pas confondre avec les échanges par contre-courant, voir plus loin) permet qu'un effet élémentaire soit amplifié le long des anses, ce qui permet d'augmenter progressivement la concentration d'un soluté jusqu'à leur point le plus profond. Les molécules de $\mathrm{NaCl}$ réabsorbées par l'épithélium du MTAL (au prix d'un transport actif, voir plus loin) peuvent entrer par diffusion à travers l'épithélium des segments grèles descendants voisins très perméables au $\mathrm{NaCl}$. Celà augmente la concentration dans le segment descendant et apporte ainsi une plus forte 
concentration de $\mathrm{NaCl}$ à la branche ascendante, qui va donc pouvoir, par itérations successives, amplifier cette différence et produire une augmentation de plus en plus forte de la concentration de $\mathrm{NaCl}$ à la pointe des anses. Ce processus permet d'expliquer l'augmentation de concentration en $\mathrm{NaCl}$ (et donc aussi d'osmolairité) observée depuis la jonction cortico-médullaire (cortex/OM) jusqu'à la jonction entre médulla externe et interne (IS/IM). Mais elle ne permet pas d'expliquer comment ce gradient progressif d'osmolarité se poursuit et même s'accroit dans la médulla interne. Ni d'expliquer comment le rein peut concentrer l'urée beaucoup plus que le sodium dans l'urine, par rapport à leurs concentrations plasmatiques respectives (voir Tableau 2 dans (3).

La Figure 8 (paneau du bas) montre les trois premières étapes de la concentration de l'urine dans un rein de mammifère simplifié. En fait, l'anse qui est dessinée semble être celle d'un néphron à anse courte, mais ce sont les néphrons à anse longue qui ont un segment grèle très perméable au $\mathrm{NaCl}(11,21)$. Donc, c'est dans les segments grèles des anses longues que pénètre le plus de $\mathrm{NaCl}$, ce qui peut en conduire une partie dans la IM. Mais il n'y a pas, en regard de ces longs DTL, de segment large ascendant pour multiplier l'effet élémentaire. Car les segments ascendants dans la IM sont des segments grèles qui ne semblent pas pouvoir assurer un transport actif significatif (peu de mitochondries, région pauvre en oxygène). Par contre, il peut y avoir des échanges par contre-courant.

II faut bien distinguer la "multiplication par contre-courant" et les "échanges par contre-courant" (21). Certes, dans les deux cas, il y a des structures disposées en parallèle et parcourues par des fluides circulant en sens inverse. Mais dans le processus de multiplication par contre-courant, il faut un "moteur" qui produit un transport actif, consommant de l'énergie, et qui peut ainsi créer une différence de concentration transépitheliale pour un soluté, en s'opposant à un gradient défavorable (dans le cas du TAL, augmenter la concentration de $\mathrm{NaCl}$ dans l'interstitium au détriment de celle de la lumière). Tandis que les échanges par contre-courant permettent seulement de limiter la dissipation d'un gradient qui a été produit ailleurs, mais pas de créer un gradient. Par exemple, l'oxygène et l'eau sont "shuntés" par des échanges par contre-courant entre les vasa recta descendants et ascendants, ce qui limite leur pénétration dans la IM. Tandis que des solutés, concentrés dans la médulla, sont "recyclés" en étant renvoyés vers la profondeur par les vasa recta artériels. Dans la IM, les solutés (en particulier l'urée, voir plus loin) peuvent subir des échanges par contre-courant entre les segments grèles ascendants et les segments grèles descendants (mais en fait un interstitium 
abondant est interposé entre ces structures et sert de pool intermédiaire). Dans les pattes des oiseaux échassiers, les calories peuvent subir un échange par contrecourant entre les artères (situées au centre des pattes) et les veines (plus périphériques) pour limiter les pertes de calories quand les oiseaux ont les pattes dans l'eau froide. Dans les reins artificiels, les solutés diffusibles subissent des échanges par contre-courant entre le circuit sanguin et le circuit du fluide de dialyse. Notez que, dans un système d'échange par contre courant, les deux tubes parallèles n'ont pas besoin d'être en continuité et peuvent même comporter des fluides différents (sang et fluide tubulaire ou fluide de dialyse).

\section{Phase 4: Concentration de l'urée dans la médulla interne}

La quatrième étape du mécanisme de concentration permet de concentrer l'urée, le soluté le plus abondant de l'urine chez les mammifères carnivores et omnivores et celui pour lequel le rein doit réabsorber la majeure partie du volume d'eau néessaire à concentrer l'urine (car d'autres solutés comme le sodium ne sont généralement pas plus concentrés dans l'urine que le plasma) (voir tableau 2 dans (3)). Chez l'homme, avec un régime alimentaire de type occidental, elle est concentrée 30 à 100 fois plus dans l'urine que dans le plasma, et chez certains rongeurs (dont rats et souris) plusieurs centaines de fois. On considère habituellement que l'urée ne fait pas l'objet d'une sécrétion active dans le rein des mammifères. Pourtant, il existe de nombreuses preuves indirectes d'une telle secrétion ainsi qu'il est expliqué dans des articles récents $(28,44,45)$. Mais peu de travaux ont été consacré à cette question dans les 20 dernières années. Cette sécrétion doit avoir lieu dans les pars recta des tubules proximaux, principalement dans la OS, et permet(trait) d'ajouter de l'urée dans la lumière du néphron au delà des quantités d'urée provenant de la filtration, comme c'est le cas pour de nombreux autres composés, azotés ou non, (acide urique, $\mathrm{NH} 4$, acides organiques, xénobiotiques). En ajoutant de l'urée dans les pars recta des néphrons à anse longue, cette sécrétion pourrait apporter des quantités d'urée significatives dans la médulla interne et augmenter le pouvoir de concentration de l'urine, comme le confirme une étude de modélisation mathématique (46).

Mais indépendamment de cette possible sécrétion, un autre mécanisme, bien démontré celui-là, permet de comprendre comment l'urée s'accumule dans la médulla interne et comment elle contribue au mécanisme de concentration (tout en en aidant surtout à sa propre concentration). Le CD est très peu permeable à l'urée 
sur la plus grande partie de sa longueur, du cortex à la partie initiale de la médulla interne, et, à la vitesse où le fluide circule, une équilibration des concentrations d'urée de part et d'autre de sa paroi n'est pas possible. Par contre, dans la partie la plus profonde de la médulla interne, la portion terminale des IMCD exprime des transporteurs facilités d'urée (UT-A1 et UT-A3), répondant à la vasopressine (Figure 9). Ils accélèrent d'un facteur 100 la vitesse de diffusion de l'urée à travers l'épithélium (47). En amont, la concentration d'urée dans la lumière a augmenté progressivement du fait de la réabsorption d'eau entraînée par la réabsorption de sodium et d'autres solutés, dans le cortex, puis dans la IS où l'accumulation de $\mathrm{NaCl}$ crée une puissante force osmotique (voir ci-dessus). La forte permeabilité à l'urée permise par ces UT-vasopressine-dépendants à la pointe de la papille permet à l'urée concentrée de diffuser dans l'interstitium environnant (igure 10). Ceci réduit l'excrétion fractionnelle d'urée mais permet d'apporter, de façon continue (en présence d'ADH) une solution concentrée d'urée dans l'interstitium de la papille.

Les vasa recta ascendants (veineux) vont entraîner cette urée vers des parties plus superficielles de la médulla où elle va subir des échanges par contre-courant avec les structures descendantes sur tout ou partie de la longueur de ces structures dans la médulla interne et externe (Figure 10). II faut préciser que les vasa recta veineux ont un endothélium fenestré qui permet une diffusion sans limite de l'urée. Les vasa recta artériels sont rendus permeables à l'urée de façon constitutive par le transporteur UT-B, et les segments grèles descendants, par UT-A2 sur une partie de leur longueur (Figure 9). Grâce à ces échanges par contre-courant, l'urée qui remontait vers la jonction cortico-médullaire dans les vasa recta ascendants est "recyclée" vers la profondeur. La fuite d'urée est minimisée et un gradient de concentration décroissante d'urée est formé, depuis la pointe de la papille jusqu'à la jonction cortico-médullaire. Et même peut-être se prolonge-t-il dans les rayons médullaires du cortex, ce qui permettrait d'expliquer les concentrations d'urée plus élevées trouvées dans le tissu cortical que dans le plasma (48). Ce gradient est maintenu tant que l'ADH agit suffisamment sur AQP2 et sur UT-A1/3 pour l' "alimenter" en apportant de l'urée concentrée à la pointe de la papille. Il s'agit d'un gradient "médullo-cortical" d'urée puisqu'il est alimenté par la partie profonde du rein et décroit progressivement vers la surface.

L'étude de souris invalidées pour les transporteurs facilités UT-A1 et UT-A3 a bien montré que l'absence de permeabilité à l'urée du CD terminal compromettait l'accumulation de l'urée dans la IM et sa concentration dans l'urine, mais pas celle du $\mathrm{NaCl}(49,50)$. En l'absence de UT-A1/3, l'excrétion d'urée est facilitée et sa 
concentration plasmatique doit s'abaisser, tandis qu'en l'absence de UT-B, le retour d'urée vers le sang périphérique est majoré, et la concentration plasmatique d'urée est augmentée (51). Dans les deux modèles, la capacité de concentration de l'urine est réduite (plus fortement toutefois avec l'invalidation de UT-A1/3 qu'avec celle d'UT-B), mais dans le cas d'UT-B, ce défaut de concentration est "urée-sélectif" car il affecte plus le rapport urine/plasma des concentrations d'urée que le rapport urine/plasma des concentrations d'osmoles totales (51). Chez l'homme, on peut aussi observer un défaut de concentration "urée-sélectif" lorsque l'architecture de la médulla est désorganisée par la présence de kystes dans la polykystose rénale, ce qui réduit les échanges par contre-courant, comme expliqué récemment (52).

II ne faut pas confondre ce "recyclage" intra-rénal d'urée (ou plutôt cette "recirculation") avec le recyclage inter-organes de l'azote uréique qui permet d'économiser l'azote en cas de nourriture pauvre en protéines. Ce dernier implique une réabsorption d'urée dans le rein, un passage dans la lumière intestinale $(53,54)$ où l'urée peut être dégradée en $\mathrm{NH}_{4}+\mathrm{CO}_{2}$ par des bactéries du microbiote exprimant l'uréase. L'azote peut alors être ré-utilisé pour former de la glutamine et retourner dans le métabolisme azoté du mammifère $(28,45)$. Ce cycle est bien développé chez les herbivores et sans doute aussi chez les ours pendant I'hibernation (55). Mais il semble n'être que très modeste chez l'homme.

Ces mouvements complexes d'urée à l'intérieur de la médulla rénale retentissent probablement sur la fonction rénale dans son ensemble car ils modifient l'osmolarité du fluide tubulaire et le rapport entre les concentrations de $\mathrm{NaCl}$ et d'urée dans les TAL. Ceci peut probablement modifier la composition du fluide tubulaire à la macula densa et donc affecter le débit de filtration glomérulaire (DFG), comme il a été expliqué dans une revue récente (56). II faut noter que, dans des études expérimentales, l'ablation chirurgicale de la papille rénale ou sa nécrose par voie chimique entraînent une baisse très significative du DFG et du poids du rein (voir tableau 3 dans (16)). D'autre part, l'augmentation du DFG observée dans la betathalassémie (57) pourrait s'expliquer par une efficacité accrue des échanges par contre-courant qui se produisent dans la médulla externe. Certes, la papille se nécrose, ce qui limite l'apport d'urée dans la médulla, mais la vitesse de circulation du sang dans les vasa recta doit être fortement ralentie par la déformation des globules rouges. Ceci augmente le temps de transit et doit améliorer l'efficacité des échanges par contre-courant dans la IS et la OS et aussi de la sécrétion d'urée qui se produit très probablement dans les pars recta (44). 


\section{Eléments indispensables pour la concentration de l'urine}

En résumé, il faut un certain nombre de facteurs, tous indispensables, pour pouvoir concentrer normalement l'urine chez les mammifères (21).

- Une disposition anatomique particulière des néphrons et des vaisseaux.

- Un "moteur" produisant un transport actif (nécessitant un apport d'énergie), capable de produire une différence de concentration transépithéliale, créeant une augmentation de la concentration de solutés dans l'interstitium environnant.

- L'expression de transporteurs ou de canaux sélectifs localisés de façon très spécifique sur des portions limitées de certains segments du néphron, du CD et des vasa recta artériels, permettant d'accélérer le transport d'eau ou le passage de certains solutés à travers les membranes cellulaires.

- Une hormone antidiurétique et des récepteurs membranaires correspondants localisés de façon très spécifique sur le $C D$.

- Un débit sanguin dans la médulla rénale suffisant pour apporter les substrats métaboliques nécessaires mais pas trop important quand même car un débit trop intense peut provoquer la dissipation du gradient d'osmoles (ou compromettre sa formation) en réduisant l'efficacité des échanges par contre courant entre vasa recta veineux et artériels.

En absence de la phase 3 (multiplication par contre courant) l'urine ne peut pas se concentrer et devient iso-osmotique au plasma (cas observé lors de l'administration d'un diurétique de l'anse). Par contre, en absence de la phase 4 (accumulation d'urée dans la médulla), l'urine peut se concentrer, mais de façon plus limitée. Enfin, en absence d'ADH, la concentration de l'urine est impossible, mais sa dilution est maintenue. Donc, l'urine est fortement hypo-osmotique.

\section{Un même segment peut avoir des fonctions en partie différentes dans le cortex et dans la médulla}

Même si certains segments du néphron et du système collecteur sont présents dans le cortex et dans la médulla (notamment le tubule proximal, le segment large ascendant et le canal collecteur), leurs fonctions dans ces différentes zones peuvent ne pas être identiques en raison de leur environnement péritubulaire différent, de la composition du fluide qui les traverse, et de certaines différences de l'épithélium qui les borde. La transition n'est pas toujours abrupte, mais elle peut être quand même 
assez nette quand les segments considéré passent d'une zone rénale à l'autre le long de leur trajet. Dans les sections suivantes, nous allons décrire quelques caractéristiques et fonctions particulières de certains segments de néphron, du canal collecteur et de l'interstitium médullaire. Nos connaissances sont surtout basées sur l'étude des reins de lapin, de rat et de souris. Les études chez l'homme ne permettent pas facilement d'analyser ces propriétés de façon différentielle, mais on peut penser que les différences observées concernent l'ensemble des mammifères, humains compris.

\section{Le tubule proximal: la pars recta versus la partie contournée}

La pars recta (PST) diffère sur certains aspects de la partie contournée du tubule proximal (PCT), comme résumé dans le Tableau 1. L'épithélium tubulaire de ces deux parties est perméable à l'eau, ce qui fait que les solutés réabsorbés ou secrétés sont suivis par des mouvements d'eau qui maintiennent l'iso-osmolarité du fluide tubulaire avec le milieu interestitiel. Mais ces deux structures ont un environnement vasculaire et tubulaire différent. Les PCT sont entourés de capillaires péritubulaires corticaux apportant du sang artériel avec un débit important. Tandis que les PST sont entourées majoritairement de vasa recta veineux remontant de zones plus profondes de la médulla (voir plus haut). D'autre part, tandis que les PCT assurent majoritairement la réabsorption de nombreux solutés présents dans le filtrat glomérulaire, les PST ont surtout des fonctions de sécrétion. C'est dans les PST que sont secretés de façon importante les acides organiques, les xénobiotiques, l'acide urique, les nucléotides. Les PST sont aussi très probablement le siège d'une intense secrétion d'urée (voir plus haut) (44). Ces sécrétions entraînent de l'eau qui suit iso-osmotiquement les solutés $(58,59)$, Chez certains poissons aglomérulaires, la sécrétion qui se produit dans le tubule proximal est à l'origine du fluide qui circule en aval dans le néphron (60). Si, chez les mammifères, elles sont quantitativement supérieures aux réabsorptions qui ont lieu simultanément dans ce même soussegment, elles vont augmenter le débit dans la branche descendante de l'anse de Henle. Les carnivores ont une fonction rénale adaptée à des repas riches en protéines, quantitativement copieux mais espacés (et donc avec des "pics" importants dans les besoins d'excrétion d'urée et autres solutés). Les tubules proximaux des carnivores possèdent, en plus des classiques segments S1, S2 et S3, un segment supplémentaire spécialisé dans les fonctions de sécrétion, avec une bordure en brosse très réduite (segment "S4") (61). Si de nombreux composés comme le cisplatine et les métaux lourds sont toxiques pour le rein, c'est souvent 
parce qu'ils pénètrent dans les pars recta où ils s'accumulent car il n'y a pas de transporteur luminal permettant de les secréter dans la lumière.

Les PST expriment des transporteurs ou canaux membranaires qui ne sont pas présents dans les PCT. En plus de l'AQP1 exprimée dans les deux sous-segments, les PST seuls expriment l'AQP7, une aquaglycéroporine, c'est à dire une aquaporine qui peut transporter de l'eau, du glycérol et de l'urée (62). Est-ce lié au métabolisme lipidique de ce segment ou à la fonction de sécrétion d'urée? D'autre part, dans le PCT, c'est le co-transporteur sodium-glucose SGLT2 sur la face apicale des cellules qui assure la réabsorption de glucose (et GLUT2 qui le laisse sortir sur la face basolatérale). Mais c'est un transporteur homologue, SGLT1 qui est majoritairement exprimé dans les PST (63). II requiert deux Na pour transporter une molécule de glucose, alors qu'il ne faut qu'un Na par glucose avec SGLT2.

En plus de ces fonctions de transport, les PST ont des fonctions métaboliques, sans doute plus intenses que les PCT (bien que peu d'études aient analysé séparemment ces deux structures). C'est dans les PST que la gluconéogénèse, à partir de lactate (un substrat produit dans la médulla et présent dans le sang des vasa recta veineux), et l'ammoniogénèse, à partir de glutamine), semblent les plus intenses (64-67). On sait que l'ammonium est un ion très toxique pour les cellules. Le fait de produire l'ammonium et de le secréter dans la lumière des PST, et non dans les PCT, permet de limiter sa diffusion dans le sang péritubulaire cortical et donc le risque d'élévation de sa concentration sanguine. Il est transporté dans les anses de Henle et est ensuite largement reabsorbé par les MTAL dans la IS puis secrété dans les canaux collecteurs voisins (68). Ce "shunt" permet d'éviter la circulation d'ammonium dans les segments corticaux du tubule distal et du canal collecteur où il risquerait de diffuser dans le sang périphérique.

Pour la gluconéogénèse, on sait que la PEPCK (phosphoenol-pyruvate carboxykinase), l'enzyme qui est le facteur limitant de cette réaction, est plus abondante dans le PST que dans le PCT (69), mais la raison de cette localisation préférentielle n'a pas été identifiée. Nous avons proposé une hypothèse nouvelle et qui s'oppose aux idées classiques. Ce glucose néoformé augmente la concentration intracellulaire en glucose et il pourrait être, en partie, secrété dans la lumière des PST par le transporteur SGLT1. Il a été montré in vitro que SGLT1 peut transporter sodium et glucose dans les deux directions (70). Le glucose pourrait ensuite diffuser hors des anses de Henle dans la médulla interne où il serait hydrolysé en lactate (qui contribue au mécanisme de concentration), participant ainsi à un cycle de Cori 
intrarénal (44). Ce glucose pourrait aussi être utilisé comme substract par la partie médullaire du TAL dans la IS. La validité de cette hypothèse n'est pas prouvée, mais elle est soutenue par plusieurs observations expérimentales et cliniques. Elle pourrait expliquer les rares cas de glycosuries d'origine rénale en absence d'hyperglycémie, lorsque le glucose secrété dans les PST ne pourrait pas être utilisé en aval.

La "natriurése de pression" est un mécanisme qui permet d'adapter l'excrétion du sodium aux besoins quotidiens malgré des régulations inappropriées qui tendent à provoquer une réabsorption sodée excessive $(23,71-73)$. Le rein étant enfermé à l'intérieur d'une capsule peu distensible, une augmentation de la pression interstitielle intra-rénale permet de freiner la réabsorption de sodium dans certains sites, dont les pars recta, et peut-être des structures médullaires plus profondes $(23,72,74)$. Donc, les pars recta jouent probablement un rôle important, sous l'effet de la pression interstitielle intrarénale, dans la régulation de la pression artérielle et de son cycle nycthéméral (75-79).

\section{Le segment large ascendant: le MTAL versus le CTAL}

On a l'habitude de parler du segment large ascendant (TAL) sans penser à distinguer la partie initiale, médullaire de ce segment, et la partie plus tardive, qui remonte dans le cortex (MTAL puis CTAL). La partie qui est dans la OS a un environnement qui ressemble plus à celui du CTAL qu'à celui du MTAL. II faut aussi noter que les CTAL des différents néphrons ont une longueur variable qui dépend de la profondeur où se trouve le glomérule du même néphron dans le cortex. Car le CTAL va venir accoler sa "macula densa" au pole vasculaire du glomérule pour former l'appareil juxtaglomérulaire. Les néphrons juxtamédullaires n'ont pratiquement pas de CTAL tandis que les néphrons les plus superficiels ont le TAL le plus long, tout au moins dans un rein de mammifère typique. Car dans le rein humain, les néphrons "corticaux" (voir plus haut), qui sont aussi les plus superficiels, ont souvent des CTAL assez courts et pas du tout de MTAL (Figure 3).

Les principales différences entre les MTAL et les CTAL sont présentées dans le Tableau 2. II existe des différences morphologiques concernant la hauteur des cellules, la richesse en mitochondries et leur association avec la membrane basolatérale (MTAL > CTAL pour toutes ces caractéristiques). Par contre les replis de la membrane basolatérale vers l'intérieur de la cellules sont plus abondants dans le CTAL. La membrane luminale ne présente partiquement pas de villosités dans le 
MTAL, mais en comporte un peu plus dans le CTAL. Le rapport entre les surfaces des membranes basolatérale et apicale est très élevé dans le MTAL (plus de 10) et plus faible dans le CTAL. Les deux sous-segments diffèrent aussi par leur équipement enzymatique (activité adénalyte cyclase et $\mathrm{Na}^{+}-\mathrm{K}^{+}$-ATPase basales environ deux fois plus élevées dans le MTAL que CTAL) (80), et les isoformes de NKCC2 $(81,82)$. Les changements morphologiques observés entre MTAL et CTAL sont graduels, mais ils sont cependant plus marqués à la hauteur de la transition entre IS et OS, c'est à dire au niveau où, autour des TAL, les pars recta des tubules proximaux se terminent pour laisser place aux segments grèles descendants. II semble plus approprié, pour diviser le TAL en deux sous-segments, de considérer que le CTAL comprend aussi la partie du TAL située dans la OS. Ces différences axiales ont été étudiées par de nombreux auteurs et ont été détaillées dans les Actualités Néphrologiques de l'Hôpital Necker en 1986 (38, 39). II faut souligner qu'il existe des différences significatives entre espèces concernant ces deux soussegments (par ex entre le lapin et le rat), sans doute en rapport avec leur régime alimentaire différent (herbivore vs rongeur) et la plus forte capacité du rat à concentrer l'urine.

Très globalement, d'un point de vue fonctionnel, on peut dire que les MTAL participent à la création du gradient osmotique de la médulla, tandis que les CTAL vont participer à la dilution de l'urine. Tous les deux réabsorbent activement du $\mathrm{NaCl}$ tout en étant pratiquement imperméables à l'eau, mais le devenir du $\mathrm{NaCl}$ réabsorbé est bien différent dans les deux zones. Dans la médulla, il sert à alimenter la multiplication par contre-courant, alors que dans le cortex, il est rapidement entrainé par l'intense débit sanguin cortical. Ces deux rôles différents expliquent bien les résultats apparemment contradictoires observés dans deux explorations fonctionnelles humaines. Pour voir si la fonction du TAL est comparable chez les noirs africains-américains et chez les caucasiens deux équipes ont employé des approches différentes. Dans l'une, une dose forte de furosémide a inhibé la fonction de transport du TAL (83), et donc la capacité de concentration de l'urine, dans l'autre, une forte charge en eau à produit une diurèse aqueuse (84). La divergence entre les conclusions des deux études vient de ce que l'une (furosémide) évaluait la capacité à re-concentrer l'urine après la disparition du gradient osmotique médullaire provoquée par le furosémide, tandis que l'autre évaluait la capacité de dilution de l'urine, sans tenir compte de la capacité de concentration. Ces études suggèrent qu'il existe sans doute des différences quantitatives dans les fonctions du TAL entre groupes ethniques (africains-américains vs caucasiens) (83-85). Ces différences peuvent retentir sur la pression artérielle. II faut noter que les concentrations 
circulantes d'AVP et la capacité de concentration de l'urine sont plus élevées chez les noirs américains que chez les caucasiens (voir revue dans (86)). II serait intéressant de faire des comparaisons semblables, à l'intérieur d'un même groupe ethnique, entre végétariens et gros consomateurs de protéines animales (à notre connaissance, les comparaisons existantes ne portent que sur le débit de filtration glomérulaire).

Du fait de son environnement hyperosmotique et de sa situation dans le système de multiplication par contre-courant, la réabsorption de $\mathrm{NaCl}$ dans le MTAL, bien qu'intense, n'entraîne qu'un abaissement assez modeste de l'osmolarité luminale (ou de concentration luminale de $\mathrm{NaCl}$ ) par rapport à l'interstitium environnant (peut-être une ou deux dizaines de mosm/L). Tandis que le CTAL a une capacité de transport plus faible (beaucoup de $\mathrm{NaCl}$ a déjà été réabsorbée en amont), mais est capable de créer une différence de concentration transépithéliale très importante. Les résultats de nombreuses expériences de microponction montrent que l'osmolarité du fluide tubulaire à la sortie des anses de Henle est environ 140-150 mosm/L alors que l'interstitium environnant est environ à 290-300 mosm/L.

Disons enfin que les adaptations fonctionnelles observées lors de divers traitements expérimentaux chroniques (variations de l'apport en sel, furosémide, intensité du mécanisme de concentration de l'urine, etc....) sont souvent différentes entre le MTAL et le CTAL. Par exemple le fait d'augmenter l'activité de concentration du rein par une perfusion chronique de dDAVP ou un régime riche en protéines provoque une hypertrophie importante du MTAL mais pas de changement ou même une réduction de l'épaisseur de l'épithélium du CTAL (comparé à la situation d'animaux ayant un faible pouvoir de concentration) (16). Associés à ces changements morphologiques dus au traitement par la dDAVP, les activités adénalyte cyclase et $\mathrm{Na}^{+}-\mathrm{K}^{+}$-ATPase par $\mathrm{mm}$ de longueur tubulaire sont augmentées dans le MTAL et diminuées dans le CTAL en réponse à une stimulation aigue par l'AVP ou le glucagon (80).

\section{Le canal collecteur: ses différents sous-segments}

Le canal collecteur n'est pas un segment du néphron. Embryologiquement, il dérive du bourgeon urétéral issu de l'ectoderme, alors que le néphron à proprement 
parler est issu du mésoderme (mésenchyme). Mais on parle souvent de "néphron distal" en incluant le canal collecteur dans cette appellation.

Les néphrons superficiels s'abouchent directement aux canaux collecteurs corticaux. Mais les néphrons profonds s'abouchent d'abord dans des tubules connecteurs qui remontent vers une partie plus superficielle du cortex avant de rejoindre les canaux collecteurs proprement dits (Figure 2). Ceci évite que les néphrons profonds rejoignent les $C D$ tout près de l'entrée de la médulla et apportent un débit de fluide trop important et possiblement encore dilué dans la médulla en échappant à la "ré-équilibration" osmotique (voir plus haut). Notez aussi que les CD ne convergent jamais dans la médulla externe (OS et IS). Ils traversent ces zones de façon rectiligne, en parallèle avec les anses de Henle, sans aucun branchement (Figure 2).

Les $C D$ traversent la totalité du rein dans son axe cortico-médullaire et ont donc des sous-segments dans le cortex et les différentes zones de la médulla. II existe de nombreuses différences anatomiques et enzymatiques, des différences d'expression de récepteurs hormonaux et de transporteurs et canaux membranaires, associées à des différences fonctionnelles entre les différentes parties du CD que nous ne pouvons énumérer ici (87-94). Nous allons seulement donner quelques informations sur des fonctions spécifiques de certains sous-segments du CD.

Du fait de l'environnement tubulaire du CD (et comme pour le TAL), et selon certaines différences anatomiques ou fonctionnelles, on peut considérer que les propriétés du CD "cortical" s'étendent aussi à la partie située dans la OS (où les pars recta voisinent avec les $C D$, comme dans les rayons médullaires). Par exemple, en réponse à un régime très pauvre en potassium, on voit la partie située dans la IS s'hypertrophier considérablement, alors que les parties situées dans la OS et le cortex restent inchangées. Certains articles décrivent que l'ENaC ou les messagers de ses sous-unités sont surtout exprimés dans le CCD et le OMCD, mais il s'agit donc sans doute plutôt du "OSCD".

On dit souvent que c'est le $C D$ qui assure la régulation finale de l'excrétion d'eau et du sodium. C'est vrai seulement en partie car des baisses de réabsorption survenues en amont peuvent dépasser ce que le CD peut compenser en aval. Par exemple, dans la natriurèse de pression, une baisse de réabsorption dans les pars recta du tubule proximal amène un flux d'eau et de sel nettement plus important au néphron distal, permettant ainsi d'excréter plus d'eau et de sel, sans que le CD 
puisse réabsorber tout l'excès qui lui est délivré. Si la réabsorption proximale baisse de $70 \%$ à $60 \%$ du filtrat (soit une baisse modeste de 14\%), et si cette différence n'est pas compensée dans le TAL et le DCT, le CD va ainsi recevoir $15 \%$ au lieu de $5 \%$ du sel filtré ( 3 fois plus). Ceci bien sûr, est juste un exemple très schématique pour donner un ordre de grandeur. Par contre, la réabsorption de sodium qui intervient dans le CD peut très fortement modifier la quantité de sodium excrétée. Si on admet que le CD reçoit à l'entrée $5 \%$ du sodium filtré et qu'il en réabsorbe $4 \%$, l'excrétion sodée est de $1 \%$ du sodium filtré. Une stimulation, même faible, de la réabsorption de sodium, passant de 4 à $4,5 \%(+11 \%)$ fait chuter l'excrétion sodée de moitié (de $1 \%$ à $0,5 \%)(40)$.

La réabsorption de sodium dans le CD cortical et médullaire externe est sous l'influence de l'aldostérone qui agit sur l'ENaC, mais, dans le rein (à la différence de l'intestin) l'aldostérone n'augmente pas l'abondance des sous-unités de l'ENaC (ou à peine pour la sous-unité alpha). La régulation rapide de la réabsorption de sodium dans cette partie du CD est influencée par l'hormone antidiurétique (par ses récepteurs V2), y compris chez l'homme $(41,95,96)$. Comme expliqué ailleurs, cette réabsorption n'a pas pour but de réguler l'excrétion sodée mais de contribuer à la conservation de l'eau en augmentant la concentration de tous les autres solutés dans la lumière du CD au détriment de celle du sodium (40). II faut cependant noter que des effets médiés par les récepteurs V1a de la vasopressine situés sur la face luminale du CD cortical, peuvent atténuer les effets V2 en stimulant (indirectement) la synthèse de prostaglandines qui accélèrent la dégradation de l'AMPc V2dépendant. II y a donc une sorte de rétro-controle des effets V2 péritubulaires par les effets V1a luminaux $(40,97,98)$. A doses fortes, la vasopressine a des effets natriurétiques probablement médiés par ces récepteurs $\mathrm{V} 1 \mathrm{a}$ (40)

L' AQP2, l'aquaporine luminale sensible à la vasopressine, est présente sur toute la longueur du canal collecteur avec une abondance qui croit le long de ce segment du cortex à la pointe de la papille (92) (bien qu'en valeur absolue, les quantités d'eau réabsorbées soient progresivement de plus en plus faibles) (43). AQP4 assure la sortie de l'eau réabsorbée sur la face basolatérale. Mais il faut signaler qu'une aquaporine supplémentaire, l'AQP3, est exprimée sur la face basolatérale des cellules du CD (en plus de AQP4) dans le cortex et la OM (sans doute au moins la $O S$ ), ce qui suppose une localisation comparable à celle de I'ENaC (mais pas sur la même membrane). Cette AQP3 est une aquaglycéroporine (perméable à l'eau, au glycérol et à l'urée). Des expériences de charge en urée chez des souris KO pour AQP3 suggèrent que cette aquaporine pourrait influencer les 
proportions respectives d'urée et de sodium dans l'urine en laissant "fuir" un peu d'urée dans le cortex, ce qui permettrait de mieux concentrer les autres solutés, dont le sodium (puisque l'eau suit l'urée lorsque l'ADH agit sur ce segment) (99). D'ailleurs, de façon intriguante, l'abondance de cette AQP3 dans le canal collecteur est fortement influencée par l'aldostérone (100), ce qui conforte l'idée qu'elle est impliquée, bien qu'indirectement, dans la régulation de l'excrétion sodée.

Dans la médulla interne, le CD comporte au moins deux sous-parties distinctes. La partie initiale, qui fait suite au CD de la médulla externe, présente une adaptation fonctionnelle très particulière quand le régime alimentaire est pauvre en protéines. Alors que le rein de la plupart des mammifères est bien adapté à une excrétion efficace d'urée (tout en économisant de l'eau), les herbivores et notamment les ruminants $(101,102)$ ont la capacité de réabsorber l'urée circulant dans le néphron, ce qui abaisse considérablement l'excrétion fractionnelle d'urée et permet de réutiliser l'azote uréique grâce à certaines bactéries du microbiote intestinal (ou du rumen) qui possèdent de l'uréase et peuvent hydroliser l'urée en $\mathrm{NH}_{4}+\mathrm{CO}_{2}$ (voir détails dans (45)). Chez ces espèces, un transporteur facilité d'urée est exprimé dans la paroi du rumen et/ou de l'intestin $(53,54,103,104)$. A partir du $\mathrm{NH}_{4}$, les bactéries peuvent synthétiser des protéines qui seront digérées et ainsi réutilisées par le mammifère, ou bien le mammifère peut synthétiser dans l'épithélium intestinal de la glutamine qui repart ensuite dans la circulation générale. Des expériences chez le rat ont montré qu'on peut induire une réabsorption active d'urée, sodiumdépendante, dans l'IMCD précoce par un régime très pauvre en protéines pendant trois semaines (105). Le transporteur responsible n'a pas (encore) été identifié (106).

L'IMCD terminal dans la partie la plus profonde de la IM possède des caractéristiques de permeabilité et de transport tout à fait spécifiques qui ont fait l'objet de nombreux travaux chez le rat et la souris. Ces canaux de la IM profonde sont très larges et ont un seul type de cellules qui ne sont pas identiques aux cellules principales des parties du CD situées en amont (cellules beaucoup plus hautes, avec un rapport entre volume du cytoplasme et noyau plus élevé, moins de mitochondries, absence de cil luminal). La lumière s'élargit fortement en approchant de l'abouchement des CD dans la cavité du pelvis, ce qui réduit la surface de contact entre fluide tubulaire et épithélium. Mais le temps de contact est sans doute augmenté car le débit du fluide diminue progressivement le long de l'IMCD au fur et à mesure que l'eau est réabsorbée. Alors que la permeabilité à l'urée est très faible tout le long du $\mathrm{CD}$, elle devient plus importante constitutivement (c'est à dire même en l'absence de vasopressine), dans l'IMCD terminal $(107,108)$. 
Les deux caractéristiques qui différencient de façon tout à fait majeure le segment terminal du IMCD de tous les sous-segments qui précèdent sont $1^{\circ}$ ) l'expression des transporteurs d'urée UT-A1 et UT-A3 sensibles à la vasopressine, qui permettent une diffusion d'urée dans l'interstitium de la partie profonde de la IM et favorisent ainsi la concentration de l'urée dans l'urine aux dépends d'une baisse de son excrétion fractionnelle (comme expliqué plus haut); et $2^{\circ}$ ) l'expression du récepteur au peptide natriurétique atrial (ANP), une hormone qui peut influencer la concentration de sodium dans l'urine, et donc la proportion entre sodium et urée, pour une même osmolarité totale.

A notre connaissance aucun travail n'a tenté de faire une étude de colocalisation de UT-A1/3 et du récepteur de l'ANP, mais il est fort probable qu'ils sont co-localisés sur les mêmes cellules. II était donc intéressant de voir si l'ANP pouvait influencer la permeabilité à l'urée de l'IMCD terminal. En utilisant la technique de microperfusion de tubule isolé par microdissection, une étude a montré que l'ANP inhibait effectivement l'augmentation de transport d'urée induite par l'ADH, de la lumière du CD vers le milieu extérieur (mais pas dans le sens opposé) $(109,110)$. II est habituellement décrit que l'ANP favorise l'excrétion du sodium en stimulant l'entrée de sodium dans la lumière de l'IMCD terminal. Mais il est probable que c'est aussi en inhibant la diffusion d'urée dans l'interstitium de la papille (111). En réduisant la concentration d'urée dans ce tissu, on permet au rapport $\mathrm{Na}$ /urée dans l'urine d'augmenter. II faut rappeler que l'ADH, par son effet sur AQP2, permet à l'osmolarité de s'équilibrer entre l'urine, dans la lumière du $C D$, et l'interstitum environnant. Donc, pour une osmolarité donnée, s'il y a une moindre concentration d'urée, il y aura une plus forte concentration de sodium (et éventuellement d'autres solutés) (voir détails dans (111).

On retrouve ici, entre ANP et ADH, et avec comme "acteur" I'IMCD terminal, une sorte de "compétition" entre urée et sodium dans la lumière du néphron, comme dans le CD cortical avec les effets de l'ENaC et de AQP3. Cette notion de "compétition" entre excrétion d'urée et de sodium n'est à notre connaissance jamais mentionnée par d'autres auteurs, tout au moins en ces termes, mais elle nous semble très importante pour la compréhension des mécanismes qui servent à économiser l'eau (en concentrant l'urée au détriment de l'excrétion du sodium) et des mécanismes qui tendent à excréter le sodium (au détriment de la conservation d'eau). Ces notions sont importantes car elles ont des conséquences sur la pression artérielle. Lorsque la balance penche en faveur de l'économie d'eau et/ou d'une 
augmentation de la sécrétion de vasopressine (chez certains sujets ou dans certaines circonstances), c'est au détriment de l'excrétion du sodium, et cette excrétion est ramenée à la normale au prix d'une augmentation de la pression artérielle.

\section{L'interstitium dans la médulla interne}

Dans la médulla interne, les structures tubulaires et les vasa recta sont entourés de cellules interstitielles (IMC = interstitial medullary cells) qui créent une isolation dans le sens radial (le long de l'axe des néphrons) et tendent ainsi à ralentir la diffusion des solutés accumulés dans l'interstitium de la médulla. Les IMC sont très riches en granules lipidiques contenant les substrats nécessaires à la synthèse de prostaglandines. Ces IMC sont faciles à isoler et à cultiver in vitro et ont fait l'objet de nombreux travaux dans les années 1970-1985. Les prostaglandines agissent en général de façon paracrine sur des structures voisines. Elles ont un rôle vasodilatateur sur la vascularisation médullaire et augmentent ainsi le débit sanguin médullaire, ce qui compromet l'accumulation de solutés dans la médulla interne (97, $98,112,113)$. Cet effet vasodilatateur est confirmé par la réduction du débit sanguin médullaire observée lors de l'inhibition de la synthèse de Pgs. Une inhibition prolongée peut d'ailleurs aboutir à une ischémie médullaire et à une nécrose papillaire, situation observée lors d'abus d'anti-inflammatoires non stéroïdiens (98). La synthèse de prostaglandines (principalement des PgE2) par les IMC est stimulée par différentes hormones, dont l'angiotensine II, la bradykinine, et surtout aussi par la vasopressine, par le biais de ses récepteurs $\mathrm{V} 1 \mathrm{a}$, abondamment exprimés par les IMC $(97,98,112,113)$. Un traitement in vivo par la vasopressine augmente l'excrétion urinaire de PgE2. D'autre part, l'effet antidiurétique de la vasopressine est potentialisé après traitement par l'indométhacine. II peut donc y avoir une certaine limite aux effets antidiurétiques de la vasopressine (et à ses effets antinatriurétiques, voir la section sur le canal collecteur), médiés par les récepteurs V2 lorsque la concentration de vasopressine s'élève de façon importante $(43,95)$.

\section{Conclusion}

En résumé, la médula rénale joue un rôle très important dans de nombreuses fonctions assurées par les reins. Les conséquences du repliement des néphrons en 
épingle à cheveux et le fait de pouvoir concentrer l'urine s'accompagnent de nombreuses adaptations qui jouent un rôle dans la physiologie du rein, mais qui peuvent aussi avoir des conséquences dans différentes pathologies. Bien sûr, on pense en premier lieu au risque de lithiase et d'infection urinaire qui augmente avec la concentration de l'urine et la réduction de la diurèse. Mais d'autres effets, moins évidents et plus indirects ont été révélés dans ces vingt dernières années. Ils dépendent de la médulla dans la mesure où ils sont associés à la concentration de l'urine et à l'axe vasopressine/hydratation. II s'agit de l'insuffisance rénale aiguë, de la polykystose rénale, de l'hypertension artérielle sensible au sel, et plus généralement de la progression de l'insuffisance rénale et de la néphropathie diabétique (comme exposé dans une revue récente (3)) (114-119). Sans oublier la récente épidémie de néphropathie méso-américaine qui pourrait être due à la déshydratation périodique des ouvriers agricoles travaillant dans des régions de fortes chaleurs (120). Pour le moment, il existe quelques études expérimentales chez l'animal qui démontrent bien ces effets pervers, mais chez l'homme, il s'agit essentiellement d'études épidémiologiques qui ne permettent pas d'établir de lien causal. La disponibilité récente d'antagonistes des récepteurs V2 de la vasopressine (les "vaptans") (121-123) pourra sans doute contribuer à mieux caractériser certains de ces effets et à peut-être à les prévenir.

\section{$\underline{\text { Liens utiles }}$}

- Des photos illustrant les relations vasculo-tubulaires dans le rein des mammifères sont disponibles sur le site internet de la Société Francophone de Néphrologie, Dialyse et Transplantation (avec légendes en français et en anglais).

- Un film d'environ 20 minutes, décrivant les relations vasculo-tubulaires dans le rein humain, est disponible sur ce même site.

- Les mêmes documents (photos et film) sont également disponibles sur le site internet "Education" de l'International Society of Nephrology.

\section{Photos avec légendes en français}

http://www.soc-nephrologie.org/epro/images/anatome.htm

http://www.theisn.org/education/education-topics/basic-science/item/1465relations-vasculo-tubulaires-dans-le-rein-des-mammiferes 
Pictures with legends in English

http://www.soc-nephrologie.org/epro/images/anatomy.htm

http://www.theisn.org/education/education-topics/basic-science/item/1464-

vascular-tubular-relationships-in-the-mammalian-kidney

Film (movie)

(commentaire en anglais/ English commentary)

http://www.soc-nephrologie.org/epro/images/humankidney/index.htm

http://www.theisn.org/education/education-topics/basic-science/item/1817-film-

the-human-kidney 


\section{Déclaration d'intérêt}

L.B. et N. B. n'ont aucun conflit d'intérêt à déclarer

\section{Remerciements}

L.B. tient à remercier les collègues qui ont joué un rôle important dans ses travaux sur l'anatomie et la physiologie rénale, et en particulier Wilhelm Kriz (Heidelberg, RFA), Brigitte Kaissling (Heidelberg, RFA, puis Bâle et Zurich, Suisse), François Morel ( $†$, Professeur au Collège de France, Paris), Christian de Rouffignac (C.E.A., Saclay), Jean-Pierre Grünfeld (Hôpital Necker, Paris), et Bodil SchmidtNielsen ( $†$,Mount Desert Island Laboratory, Maine, USA).

\section{Legendes des figures}

Figure 1. Haut. Coupe de rein de rat montrant la vascularisation artérielle grâce à l'injection de silastic (Microfil silicone rubber@) dans l'artère rénale. On distingue bien les quatre zones rénales: cortex (C), zones externe (OS) et interne (IS) de la médulla externe, et médulla interne (IM). Dans le cortex superficiel et moyen, on distingue bien les glomérules et un riche réseau capillaire entourant les tubules (non visibles ici). Dans le cortex profond, noter l'alternance entre des zones "labyrinthiques", richement capillarisées comme le reste du cortex, et les rayons médullaires du cortex (dépouvus de glomérules) formant des cônes plus sombres. Ces rayons médullaires ainsi que toute la OS reçoivent peu de capillaires et sont alimentés surtout par des vasa recta veineux remontant de la médulla plus profonde (non visibles ici, dans cette injection artérielle). Dans la IM, la résistance dans les vasa recta est élevée (du fait de leur grande longueur) et ils n'ont pas été très remplis. En cas de vasodilatation de la circulation médullaire, on peut observer une densité vasculaire bien plus considérable dans la IM. Figure 1. Bas. Coupe de rein de rat après coloration in vivo par une perfusion intraveineuse de bleu Alcian. Ce colorant se fixe sur les mucopolysaccharides de la paroi endothéliale. Les floculus des glomérules sont très fortement marqués. Dans la médulla, les zones colorées (en gris foncé ici) sont $1^{\circ}$ ) la zone interne de la 
médulla externe (IS) en raison de la densité vasculaire relativement intense autour des TAL et $C D$ de cette région, et $2^{\circ}$ ) la papille, à l'extrémité de la médulla interne (IM) probablement en raison du fait que l'endothélium des vasa recta est fortement fenestré et laisse ainsi s'extravaser le colorant.

Figure 2. Schéma montrant un néphron à anse courte et un néphron à anse longue se raccordant à un canal collecteur, tels qu'on les observe chez un mammifère typique. Les 4 zones rénales sont clairement délimitées par la présence des glomérules (exclusivement dans le cortex) et les transitions entres les différents segments du néphron: limite OS/IS à la transition entre pars recta et segment grèle; limite IS/IM à la transition entre segment grèle et segment large ascendant. Dans la zone corticale, les rayons médullaires (MR) sont une sorte d'extension de la OS dans le cortex profond. Sur ce schéma, la partie contournée des tubules proximaux (PCT) est figurée ici avec des petits points, tandis que la pars recta (PST) est colorée en bleu. La partie médullaire des segments larges ascendants (MTAL) est colorée en rouge et la partie corticale (CTAL) (pratiquement absente dans les néphrons à anse longue) est hachurée. Les tubules contournés distaux (DCT) sont colorés en noir. Sur le canal collecteur terminal de la médulla interne (IMCD), la partie bordée de vert est celle où les transporteurs facilités d'urée vasopressine-sensibles, UT-A1 et UT-A3, sont exprimés. Pour les abréviations des zones rénales et des autres segments du néphron et du canal collecteur, voir la liste des abréviations au début de l'article. Adapté de (38)

Figure 3. Schéma montrant les différents types de néphrons et leurs longueurs respectives dans le rein des rongeurs et dans le rein humain. Les tubules proximaux sont en couleur claire et les TAL et les glomérules sont représentés en noir. Dans le rein des rongeurs, tous les néphrons à anse courte "tournent" à la même profondeur dans la médulla, tandis que dans le rein humain, on observe une plus grande variabilité et une proportion importante de néphrons "corticaux" dont l'anse est très courte, dépouvue de segment grèle et ne pénètre pas du tout dans la médulla. Le rein humain a une proportion de néphrons à anse longue plus faible que chez les rongueurs. Les castors n'ont pratiquement pas de médulla interne ni donc de néphrons à anse longue. Par contre, les carnivores (non représentés) ont $100 \%$ de néphrons à anse longue. Tous leurs néphrons pénètrent au moins sur une certaine longeur dans la médulla interne. Adapté de (35). 
Figure 4. Schéma montrant la longeur des anses de Henle de 70 néphrons microinjectés avec du silastic par une micropipette insérée dans l'espace urinaire du glomérule. Le haut de chaque ligne montre l'emplacement du glomérule dans l'épaisseur du cortex et le bas, la pointe de l'anse de Henle du même néphron (toutes les distances ont été normalisées par rapport à une longueur standard entre surface du cortex et extrémité de la médulla). Les lignes horizontales montrent les limites des zones rénales. Données aimablement fournies par le $\mathrm{Dr}$ Reiner Beeuwkes, Boston, USA et reproduites avec son autorisation.

Figure 5. Coupe longitudinale de rein de rat dont la vascularisation artérielle a été visualisée par du silastic (Microfil silicone rubber@) injecté dans l'artère rénale centrée sur la IS. La zone arquée richement vascularisée est la zone interne (IS) de la médulla externe. Le cortex profond est visible en haut de la photo. Les artérioles efférentes des glomérules profonds se divisent en bouquets de vasa recta descendants dans la médulla sans donner de tributaires dans la OS (zone presque dépourvue de capillaires artériels). Les vaisseaux veineux ne sont pas visibles sur cette préparation. Dans la IS, on distingue bien l'alternance entre des faisceaux vasculaires contenant des vaisseaux relativement rectilignes, les vasa recta, et des zones interfasciculaire riches en capillaires. Ces réseaux capillaires s'interrompent brusquement à la jonction entre médulla externe et médulla interne, là où se trouve la transition entre segments grèles et segments larges ascendants. Ceci crée ainsi une bonne isolation entre ces deux zones. Dans la IS, des vasa recta veineux ascendants (non visibles ici) sont intimement associés aux vasa recta artériels dans les faisceaux vasculaires. La communication entre IS et IM passe uniquement par les vasa recta, mais les échanges par contre-courant entre sang artériel et veineux favorisent les échanges "horizontaux" et limitent les échanges dans le sens "vertical" (dans l'axe des néphrons). Sur cette préparation, les vasa recta de la médulla interne n'ont pas été remplis par le silastic (à part quelques uns) car l'injection n'a pas été poussée suffisemment pour vaincre la résistance élevée dans ces vaisseaux.

Figure 6. A gauche et au milieu. Coupes de rein de lapin, centrées sur la OS, et montrant à gauche, la vascularisation artérielle (silastic injecté par l'artère rénale) et au milieu, la vascularisation veineuse (silastic injecté de façon rétrograde par la veine rénale). Noter les artères et veines interlobulaires dans le cortex. Sur la photo du milieu, on remarque l'abondance de la vascularisation veineuse dans la OS, formée par des vasa recta qui remontent des régions plus profondes de la médulla. Figure 6. A droite. Coupe histologique transversale de rein de rat au 
niveau de la OS. On distingue un grand nombre de pars recta $(\mathrm{Pr})$ reconnaissables à leur bordure en brosse et leurs vésicules sous luminales, des TAL (d) et des canaux collecteurs (C). Entre ces structures se trouvent de très nombreux vasa recta veineux (V) qui offrent une grande surface de contact avec les tubules. Reproduit de (28).

Figure 7. Autoradiographies révélant les sites de fixation de différentes hormones marquées à l'iode ${ }^{125}$ : l'angiotensine II (All), la dDAVP (un agoniste peptidique sélectif des récepteurs V2 de la vasopressine) (AVP-R2), le peptide natriurétique atrial (ANP), et l'endothéline (ET), sur des coupes de rein de rat. II s'agit de fausses couleurs; le rouge indique le marquage le plus intense et le bleu l'absence de marquage (niveau du bruit de fond). La fixation non-spécifique (non présentée) est négligeable. On ne peut pas comparer quantitativement les différentes photos entre elles car le marquage radioactif du ligand et les temps de pose des autoradiographies ont pu être différents d'une expérience à l'autre. La papille paraît tronquée dans certaines photos car les coupes de rein n'ont pas toujours pu être orientées exactement dans l'axe cortico-papillaire. Photos pour All, ANP et ET réalisées par Frederick Mendelsohn (Université de Melbourne, Australie), reproduites avec sa permission. Photo pour l'AVP-R2 reproduite à partir de la couverture de Kidney International (Volume 76(2), 2009).

Figure 8. Schéma permettant de comparer un néphron d'amphibien et un néphron (simplifié) de mammifère. Ce schéma montre les trois étapes: dilution, équilibration et concentration, ainsi que les structures où agit l'hormone antidiurétique (vasotocine ou vasopressine). Le TAL des mammifères a des propriétés de transport équivalentes à celles du segment de dilution des amphibiens. Chez les amphibiens, la vasotocine agit non seulement sur le canal collecteur, mais aussi sur la vessie. Le néphron du mammifère est représenté ici avec une anse courte par simplicité, mais en fait, ce sont surtout les néphrons à anse longue qui participent à la multiplication par contre-courant.

Figure 9. Hybridation in situ des trois principaux transporteurs facilités d'urée exprimés dans le rein de rat. UT-A1/A3 est strictement limité aux canaux collecteurs terminaux à la pointe de la papille. UT-A2 est exprimé dans la moitié la plus profonde des segments grèles descendants des néphrons à anse courte, tandis que AQP1 (non visible ici) est exprimé dans la partie initiale de ces segments (en continuité avec les pars recta). UT-B est exprimé sur l'endothélium des vasa recta artériels (descendants) dans toute la IS et la IM. II est aussi 
présent dans l'épithélium qui borde le pelvis. II n'y a pas de transporteur facilité d'urée dans le cortex ni dans la OS. La barre noire représente $2 \mathrm{~mm}$. Reproduit de (124).

Figure 10. Recirculation intra-rénale d'urée participant à se concentration dans I'urine. L'eau réabsorbée le long du CD permet de concentrer l'urée dans la lumière. Des transporteurs facilités d'urée (UT-A1/3) sont exprimés uniquement dans la partie terminale de l'IMCD, ce qui permet à l'urée, concentrée en amont, de diffuser dans l'interstitium de la IM. L'urée tend ensuite continuellement à s'échapper de cette région par les vasa recta ascendants veineux (AVR, en bleu), mais une proportion variable est ramenée à la IM pas des échanges par contrecourant entre vasa recta ascendants et descendants (DVR, en rouge), échanges plus particulièrements intenses dans les faisceaux vasculaires situés dans la IS. La sécrétion active d'urée par la pars recta (hypothèse très fortement plausible) dans la OS apporte un supplément d'urée dans la lumière du néphron dont une partie peut diffuser dans la IM et une partie circuler dans le CD et alimenter la recirculation décrite plus haut. La désorganisation de la médulla par des kystes peut compromettre l'efficacité de cette recirculation d'urée. Des prostaglandines produites par les cellules interstitielles de la IM (i.m.c.) peuvent vasodilater la vascularisation médullaire et réduire l'efficacité de cette recirculation. Reproduit de (52) 


\section{Références}

1. Kriz W, Bankir L. A standard nomenclature for structures of the kidney. The Renal Commission of the International Union of Physiological Sciences (IUPS). Kidney Int. 1988;33(1):1-7.

2. Kriz W, Bankir L. A standard nomenclature for structures of the kidney. The Renal Commission of the International Union of Physiological Sciences (IUPS). The American journal of physiology. 1988;254(1 Pt 2):F1-8.

3. Bankir L, Bouby N, Ritz E. Vasopressin: a novel target for the prevention and retardation of kidney disease? Nature reviews Nephrology. 2013;9(4):223-39.

4. Kriz W. Structural organization of the renal medulla: comparative and functional aspects. Am J Physiol. 1981;241(1):R3-16.

5. Kriz W. Structural organization of the renal medullary counterflow system. Fed Proc. 1983;42(8):2379-85.

6. Kriz W, Kaissling B. Structural Organization of the Mammalian Kidney. In: Seldin D, Giebisch G, editors. The Kidney. 1: Elsevier; 2008. p. 479-563.

7. Christensen EI, Wagner CA, Kaissling B. Uriniferous tubule: structural and functional organization. Compr Physiol. 2012;2(2):805-61.

8. Song R, Yosypiv IV. Development of the kidney medulla. Organogenesis. 2012;8(1):10-7.

9. Bankir L, Bouby N, Trinh-Trang-Tan MM. Heterogeneity of nephron anatomy. Kidney Int Suppl. 1987;20:S25-39.

10. Williams MF. Morphological evidence of marine adaptations in human kidneys. Med Hypotheses. 2006;66(2):247-57.

11. Bankir L, de Rouffignac C. Urinary concentrating ability: insights from comparative anatomy. Am J Physiol. 1985;249(6 Pt 2):R643-66.

12. Beuchat CA. Structure and concentrating ability of the mammalian kidney: correlations with habitat. Am J Physiol. 1996;271(1 Pt 2):R157-79.

13. Sperber I. Studies on the mammalian kidney. Zool Bidr Uppsala. 1944;22:249432.

14. Beeuwkes R, 3rd. The vascular organization of the kidney. Annu Rev Physiol. 1980;42:531-42.

15. Bankir L, Kaissling B, de Rouffignac $C$, Kriz W. The vascular organization of the kidney of Psammomys obesus. Anat Embryol (Berl). 1979;155(2):149-60.

16. Bankir L, Kriz W. Adaptation of the kidney to protein intake and to urine concentrating activity: similar consequences in health and CRF. Kidney Int. 1995;47(1):7-24.

17. Elger M, Bankir L, Kriz W. Morphometric analysis of kidney hypertrophy in rats after chronic potassium depletion. Am J Physiol. 1992;262(4 Pt 2):F656-67.

18. Bankir L, Fischer C, Fischer S, Jukkala K, Specht HC, Kriz W. Adaptation of the rat kidney to altered water intake and urine concentration. Pflugers Arch. 1988;412(1-2):42-53.

19. Bouby N, Bankir L, Trinh-Trang-Tan MM, Minuth WW, Kriz W. Selective ADHinduced hypertrophy of the medullary thick ascending limb in Brattleboro rats. Kidney Int. 1985;28(3):456-66. 
20. Bouby N, Trinh-Trang-Tan MM, Laouari D, Kleinknecht C, Grunfeld JP, Kriz W, et al. Role of the urinary concentrating process in the renal effects of high protein intake. Kidney Int. 1988;34(1):4-12.

21. Bankir L, Bouby N, Trinh-Trang-Tan MM. The role of the kidney in the maintenance of water balance. Baillieres Clin Endocrinol Metab. 1989;3(2):249311.

22. Pallone TL, Edwards A, Mattson DL. Renal medullary circulation. Compr Physiol. 2012;2(1):97-140.

23. O'Connor PM, Cowley AW, Jr. Modulation of pressure-natriuresis by renal medullary reactive oxygen species and nitric oxide. Curr Hypertens Rep. 2010;12(2):86-92.

24. Levillain O, Hus-Citharel A, Morel F, Bankir L. Localization of arginine synthesis along rat nephron. Am J Physiol. 1990;259(6 Pt 2):F916-23.

25. Levillain O, Hus-Citharel A, Morel F, Bankir L. Arginine synthesis in mouse and rabbit nephron: localization and functional significance. Am J Physiol. 1993;264(6 Pt 2):F1038-45.

26. Levillain O, Hus-Citharel A, Morel F, Bankir $L$. Production of urea from arginine in pars recta and collecting duct of the rat kidney. Ren Physiol Biochem. 1989;12(5-6):302-12.

27. Levillain O, Hus-Citharel A, Morel F, Bankir L. Localization of urea and ornithine production along mouse and rabbit nephrons: functional significance. Am J Physiol. 1992;263(5 Pt 2):F878-85.

28. Bankir L, Trinh-Trang-Tan MM. Urea and the kidney. In: Brenner BM, editor. The Kidney (6th Edition). Philadelphia: W B Saunders Company; 2000. p. 63779.

29. Johnson RJ, Rodriguez-lturbe B, Roncal-Jimenez C, Lanaspa MA, Ishimoto T, Nakagawa $T$, et al. Hyperosmolarity drives hypertension and CKD--water and salt revisited. Nat Rev Nephrol. 2014;10(7):415-20.

30. Zhuo J, Song K, Harris PJ, Mendelsohn FA. In vitro autoradiography reveals predominantly AT1 angiotensin II receptors in rat kidney. Ren Physiol Biochem. 1992;15(5):231-9.

31. Sexton PM, Zhuo J, Mendelsohn FA. Localization and regulation of renal receptors for angiotensin II and atrial natriuretic peptide. Tohoku J Exp Med. 1992;166(1):41-56.

32. Kohzuki M, Johnston Cl, Chai SY, Casley DJ, Mendelsohn FA. Localization of endothelin receptors in rat kidney. Eur J Pharmacol. 1989;160(1):193-4.

33. de Rouffignac C, Corman B, Roinel N. Stimulation by antidiuretic hormone of electrolyte tubular reabsorption in rat kidney. Am J Physiol. 1983;244(2):F15664.

34. De Rouffignac C, Di Stefano A, Wittner M, Roinel N, Elalouf JM. Consequences of differential effects of $A D H$ and other peptide hormones on thick ascending limb of mammalian kidney. The American journal of physiology. 1991;260(6 Pt 2):R1023-35.

35. Bankir L. La fonction de dilution de l'urine. Ann Med Nancy. 1991;30:19-25.

36. Berl T. Impact of solute intake on urine flow and water excretion. J Am Soc Nephrol. 2008;19(6):1076-8.

37. Burg MB. Thick ascending limb of Henle's loop. Kidney Int. 1982;22(5):454-64.

38. Bankir L, Bouby N, Trinh-Trang-Tan MM, Kaissling B. La branche large ascendante de l'anse de Henle : caractéristiques anatomiques et fonctionnelles et rôle dans le mécanisme de concentration de l'urine. In: Grünfeld JP, editor. 
Actualités Néphrologiques de l'Hôpital Necker: Flammarion Médecine-Sciences; 1986. p. 66-96.

39. Bankir L, Bouby N, Trinh-Trang-Tan MM, Kaissling B. Thick ascending limb-anatomy and function: role in urine concentrating mechanisms. Adv Nephrol Necker Hosp. 1987;16:69-102.

40. Bankir L, Bichet DG, Bouby N. Vasopressin V2 receptors, ENaC, and sodium reabsorption: a risk factor for hypertension? Am J Physiol Renal Physiol. 2010;299(5):F917-28.

41. Bankir L, Fernandes S, Bardoux P, Bouby N, Bichet DG. Vasopressin-V2 receptor stimulation reduces sodium excretion in healthy humans. J Am Soc Nephrol. 2005;16(7):1920-8.

42. Schafer JA. The rat cortical collecting duct as an isosmotic volume reabsorber. In: Ussing $\mathrm{HH}$, Fischbarg J, Sten-Knudsen $\mathrm{O}$, Larsen $\mathrm{FH}$, Willumsen $\mathrm{NJ}$, editors. Isotonic transport in leaky epithelia. Copenhagen: Alfred Benzon Symposium; 1993. p. 339-50.

43. Bankir L. Antidiuretic action of vasopressin: quantitative aspects and interaction between V1a and V2 receptor-mediated effects. Cardiovascular research. 2001;51(3):372-90.

44. Bankir L, Yang B. New insights into urea and glucose handling by the kidney, and the urine concentrating mechanism. Kidney international. 2012;81(12):1179-98.

45. Bankir L. Active urea transport in lower vertebrates and mammals. Subcell Biochem. 2014;73:193-226.

46. Layton AT, Bankir L. Impacts of Active Urea Secretion into Pars Recta on Urine Concentration and Urea Excretion Rate. Physiological reports. 2013;1(3) e00034.

47. Fenton RA. Urea transporters and renal function: lessons from knockout mice. Current opinion in nephrology and hypertension. 2008;17(5):513-8.

48. Roch-Ramel F, Chomety F, Peters G. Urea concentrations in tubular fluid and in renal tissue of nondiuretic rats. Am J Physiol. 1968;215(2):429-38.

49. Fenton RA, Chou CL, Stewart GS, Smith CP, Knepper MA. Urinary concentrating defect in mice with selective deletion of phloretin-sensitive urea transporters in the renal collecting duct. Proc Natl Acad Sci U S A. 2004;101(19):7469-74.

50. Fenton RA, Flynn A, Shodeinde A, Smith CP, Schnermann J, Knepper MA. Renal phenotype of UT-A urea transporter knockout mice. J Am Soc Nephrol. 2005;16(6):1583-92.

51. Yang B, Bankir L. Urea and urine concentrating ability: new insights from studies in mice. American journal of physiology Renal physiology. 2005;288(5):F881-96.

52. Bankir L, Bichet DG. Polycystic kidney disease: An early urea-selective urineconcentrating defect in ADPKD. Nat Rev Nephrol. 2012;8(8):437-9.

53. Simmons NL, Chaudhry AS, Graham C, Scriven ES, Thistlethwaite A, Smith $\mathrm{CP}$, et al. Dietary regulation of ruminal bovine UT-B urea transporter expression and localization. J Anim Sci. 2009;87(10):3288-99.

54. Stewart GS, Graham C, Cattell S, Smith TP, Simmons NL, Smith CP. UT-B is expressed in bovine rumen: potential role in ruminal urea transport. Am J Physiol Regul Integr Comp Physiol. 2005;289(2):R605-R12. 
55. Stenvinkel P, Frobert O, Anderstam B, Palm F, Eriksson M, Bragfors-Helin AC, et al. Metabolic changes in summer active and anuric hibernating free-ranging brown bears (Ursus arctos). PLoS One. 2013;8(9):e72934.

56. Bankir L, Roussel R, Bouby N. Protein- and diabetes-induced glomerular hyperfiltration: role of glucagon, vasopressin, and urea. Am J Physiol Renal Physiol. 2015;309(1):F2-23.

57. Becker AM. Sickle cell nephropathy: challenging the conventional wisdom. Pediatr Nephrol. 2011;26(12):2099-109.

58. Grantham JJ, Irwin RL, Qualizza PB, Tucker DR, Whittier FC. Fluid secretion in isolated proximal straight renal tubules. Effect of human uremic serum. J Clin Invest. 1973;52(10):2441-50.

59. Grantham JJ, Qualizza PB, Irwin RL. Net fluid secretion in proximal straight renal tubules in vitro: role of PAH. Am J Physiol. 1974;226(1):191-7.

60. Beyenbach KW. Kidneys sans glomeruli. Am J Physiol Renal Physiol. 2004;286(5):F811-27.

61. Bulger RE, Cronin RE, Dobyan DC. Survey of the morphology of the dog kidney. Anat Rec. 1979;194:41-66.

62. Sohara E, Uchida S, Sasaki S. Function of aquaporin-7 in the kidney and the male reproductive system. Handb Exp Pharmacol. 2009(190):219-31.

63. Wright EM, Hirayama BA, Loo DF. Active sugar transport in health and disease. J Intern Med. 2007;261(1):32-43.

64. Maleque A, Endou H, Koseki C, Sakai F. Nephron heterogeneity: gluconeogenesis from pyruvate in rabbit nephron. FEBS Lett. 1980;116(2):1546.

65. Conjard A, Martin M, Guitton J, Baverel G, Ferrier B. Gluconeogenesis from glutamine and lactate in the isolated human renal proximal tubule: longitudinal heterogeneity and lack of response to adrenaline. Biochem J. 2001;360(Pt 2):371-7.

66. Curthoys NP, Moe OW. Proximal tubule function and response to acidosis. Clin J Am Soc Nephrol. 2014;9(9):1627-38.

67. Weiner ID, Verlander JW. Renal ammonia metabolism and transport. Compr Physiol. 2013;3(1):201-20.

68. Karim Z, Szutkowska M, Vernimmen C, Bichara M. Recent concepts concerning the renal handling of $\mathrm{NH} 3 / \mathrm{NH} 4+$. J Nephrol. 2006;19 Suppl 9:S27-32.

69. Drewnowsk KD, Craig MR, Digiovanni SR, McCarty JM, Moorman AF, Lamers $\mathrm{WH}$, et al. PEPCK mRNA localization in proximal tubule and gene regulation during metabolic acidosis. J Physiol Pharmacol. 2002;53(1):3-20.

70. Eskandari S, Wright EM, Loo DD. Kinetics of the reverse mode of the $\mathrm{Na}+$ /glucose cotransporter. J Membr Biol. 2005;204(1):23-32.

71. Hall JE, Guyton AC, Coleman TG, Mizelle HL, Woods LL. Regulation of arterial pressure: role of pressure natriuresis and diuresis. Fed Proc. 1986;45(13):2897903.

72. Evans RG, Majid DS, Eppel GA. Mechanisms mediating pressure natriuresis: what we know and what we need to find out. Clin Exp Pharmacol Physiol. 2005;32(5-6):400-9.

73. Ivy JR, Bailey MA. Pressure natriuresis and the renal control of arterial blood pressure. J Physiol. 2014;592(Pt 18):3955-67.

74. Granger JP, Alexander BT, Llinas M. Mechanisms of pressure natriuresis. Curr Hypertens Rep. 2002;4(2):152-9. 
75. Bankir L, Bochud M, Maillard M, Bovet P, Gabriel A, Burnier M. Nighttime blood pressure and nocturnal dipping are associated with daytime urinary sodium excretion in African subjects. Hypertension. 2008;51(4):891-8.

76. Burnier M, Coltamai L, Maillard M, Bochud M. Renal sodium handling and nighttime blood pressure. Semin Nephrol. 2007;27(5):565-71.

77. Fujii T, Uzu T, Nishimura M, Takeji M, Kuroda S, Nakamura S, et al. Circadian rhythm of natriuresis is disturbed in nondipper type of essential hypertension. Am J Kidney Dis. 1999;33(1):29-35.

78. Uzu T, Kimura G. Diuretics shift circadian rhythm of blood pressure from nondipper to dipper in essential hypertension. Circulation. 1999;100(15):1635-8.

79. Fukuda M, Uzu T, Kimura G. Duration until nighttime blood pressure fall indicates excess sodium retention. Chronobiol Int. 2012;29(10):1412-7.

80. Trinh-Trang-Tan MM, Bankir L, Doucet A, el Mernissi G, Imbert-Teboul M, Montegut $\mathrm{M}$, et al. Influence of chronic $\mathrm{ADH}$ treatment on adenylate cyclase and ATPase activity in distal nephron segments of diabetes insipidus Brattleboro rats. Pflugers Archiv : European journal of physiology. 1985;405(3):216-22.

81. Carota I, Theilig F, Oppermann M, Kongsuphol P, Rosenauer A, Schreiber R, et al. Localization and functional characterization of the human NKCC2 isoforms. Acta Physiol (Oxf). 2010;199(3):327-38.

82. Castrop H, Schnermann J. Isoforms of renal Na-K-2Cl cotransporter NKCC2: expression and functional significance. Am $J$ Physiol Renal Physiol. 2008;295(4):F859-66.

83. Chun TY, Bankir L, Eckert GJ, Bichet DG, Saha C, Zaidi SA, et al. Ethnic differences in renal responses to furosemide. Hypertension. 2008;52(2):241-8.

84. Weder AB, Gleiberman L, Sachdeva A. Whites excrete a water load more rapidly than blacks. Hypertension. 2009;53(4):715-8.

85. Bankir L, Perucca J, Weinberger $\mathrm{MH}$. Ethnic differences in urine concentration: possible relationship to blood pressure. Clin J Am Soc Nephrol. 2007;2(2):30412.

86. Perucca J, Bouby N, Valeix P, Jungers $P$, Bankir L. Différence de concentration urinaire selon le sexe ou l'origine ethnique : implications possibles dans la susceptibilité variable à différentes pathologies rénales et cardiovasculaires [Difference in urine concentration according to gender and ethnicity: possible involvement in the different susceptibility to various renal and cardiovascular diseases]. Nephrol Ther. 2008;4(3):160-72.

87. Brown EM, Pollak M, Hebert SC. The extracellular calcium-sensing receptor: its role in health and disease. Annu Rev Med. 1998;49:15-29.

88. Carmosino M, Brooks HL, Cai Q, Davis LS, Opalenik S, Hao C, et al. Axial heterogeneity of vasopressin-receptor subtypes along the human and mouse collecting duct. Am J Physiol Renal Physiol. 2007;292(1):F351-60.

89. Christensen BM, Wang W, Frokiaer J, Nielsen S. Axial heterogeneity in basolateral AQP2 localization in rat kidney: effect of vasopressin. Am J Physiol Renal Physiol. 2003;284(4):F701-17.

90. LeFurgey A, Tisher CC. Morphology of rabbit collecting duct. Am J Anat. 1979;155(1):111-24.

91. Lombard WE, Kokko JP, Jacobson HR. Bicarbonate transport in cortical and outer medullary collecting tubules. Am J Physiol. 1983;244(3):F289-96. 
92. Kishore BK, Terris JM, Knepper MA. Quantitation of aquaporin-2 abundance in microdissected collecting ducts: axial distribution and control by AVP. Am J Physiol. 1996;271(1 Pt 2):F62-70.

93. Lynch IJ, Greenlee MM, Gumz ML, Rudin A, Xia SL, Wingo CS. Heterogeneity of $\mathrm{H}-\mathrm{K}-\mathrm{ATPase}$-mediated acid secretion along the mouse collecting duct. Am J Physiol Renal Physiol. 2010;298(2):F408-15.

94. Ridderstrale Y, Kashgarian M, Koeppen B, Giebisch G, Stetson D, Ardito T, et al. Morphological heterogeneity of the rabbit collecting duct. Kidney Int. 1988;34(5):655-70.

95. Perucca J, Bichet DG, Bardoux P, Bouby N, Bankir L. Sodium excretion in response to vasopressin and selective vasopressin receptor antagonists. Journal of the American Society of Nephrology : JASN. 2008;19(9):1721-31.

96. Blanchard A, Frank M, Wuerzner G, Peyrard S, Bankir L, Jeunemaitre X, et al. Antinatriuretic effect of vasopressin in humans is amiloride sensitive, thus $\mathrm{ENaC}$ dependent. Clin J Am Soc Nephrol. 2011;6(4):753-9.

97. Hao CM, Breyer MD. Physiological regulation of prostaglandins in the kidney. Annu Rev Physiol. 2008;70:357-77.

98. Schlondorff D. Renal prostaglandin synthesis. Sites of production and specific actions of prostaglandins. Am J Med. 1986;81(2b):1-11.

99. Zhao D, Bankir L, Qian L, Yang D, Yang B. Urea and urine concentrating ability in mice lacking AQP1 and AQP3. Am $J$ Physiol Renal Physiol. 2006;291(2):F429-38.

100. Kwon TH, Nielsen J, Masilamani S, Hager H, Knepper MA, Frokiaer J, et al. Regulation of collecting duct AQP3 expression: response to mineralocorticoid. Am J Physiol Renal Physiol. 2002;283(6):F1403-21.

101. Schmidt-Nielsen $B$, Osaki $H$. Renal response to changes in nitrogen metabolism in sheep. Am J Physiol. 1958;193(3):657-61.

102. Schmidt-Nielsen B, Osaki H, Murdaugh HV, Jr., O'Dell R. Renal regulation of urea excretion in sheep. Am J Physiol. 1958;194(2):221-8.

103. Marini JC, Klein JD, Sands JM, Van Amburgh ME. Effect of nitrogen intake on nitrogen recycling and urea transporter abundance in lambs. J Anim Sci. 2004;82(4):1157-64.

104. Reynolds CK, Kristensen NB. Nitrogen recycling through the gut and the nitrogen economy of ruminants: an asynchronous symbiosis. J Anim Sci. 2008;86(14 Suppl):E293-305.

105. Isozaki T, Lea JP, Tumlin JA, Sands JM. Sodium-dependent net urea transport in rat initial inner medullary collecting ducts. J Clin Invest. 1994;94(4):1513-7.

106. Sands JM, Martial S, Isozaki T. Active urea transport in the rat inner medullary collecting duct: functional characterization and initial expression cloning. Kidney Int. 1996;49(6):1611-4.

107. Sands JM, Knepper MA. Urea permeability of mammalian inner medullary collecting duct system and papillary surface epithelium. J Clin Invest. 1987;79(1):138-47.

108. Sands JM, Nonoguchi $\mathrm{H}$, Knepper MA. Vasopressin effects on urea and $\mathrm{H} 2 \mathrm{O}$ transport in inner medullary collecting duct subsegments. Am J Physiol. 1987;253(5 Pt 2):F823-32.

109. Rocha AS, Kudo LH. Atrial peptide and cGMP effects on $\mathrm{NaCl}$ transport in inner medullary collecting duct. Am J Physiol. 1990;259(2 Pt 2):F258-68. 
110. Rocha AS, Kudo LH. Effect of atrial natriuretic factor and cyclic guanosine monophosphate on water and urea transport in the inner medullary collecting duct. Pflugers Arch. 1990;417(1):84-90.

111. Bankir LT, Trinh-Trang-Tan MM. Renal urea transporters. Direct and indirect regulation by vasopressin. Exp Physiol. 2000;85 Spec No:243s-52s.

112. Zhuo JL. Renomedullary interstitial cells: a target for endocrine and paracrine actions of vasoactive peptides in the renal medulla. Clin Exp Pharmacol Physiol. 2000;27(7):465-73.

113. Nørregaard R, Kwon TH, Frøkiaer J. Physiology and pathophysiology of COX-2 and PGE2 in the kidney Kidney Reseach Clinical Practice. 2015;34:194-200.

114. Clark WF, Sontrop JM, Macnab JJ, Suri RS, Moist L, Salvadori M, et al. Urine volume and change in estimated GFR in a community-based cohort study. Clin J Am Soc Nephrol. 2011;6(11):2634-41.

115. Boertien WE, Meijer E, Zittema D, van Dijk MA, Rabelink TJ, Breuning MH, et al. Copeptin, a surrogate marker for vasopressin, is associated with kidney function decline in subjects with autosomal dominant polycystic kidney disease. Nephrol Dial Transplant. 2012;27(11):4131-7.

116. Meijer E, Bakker SJ, de Jong PE, Homan van der Heide JJ, van Son WJ, Struck $\mathrm{J}$, et al. Copeptin, a surrogate marker of vasopressin, is associated with accelerated renal function decline in renal transplant recipients. Transplantation. 2009;88(4):561-7.

117. Riphagen IJ, Boertien WE, Alkhalaf A, Kleefstra N, Gansevoort RT, Groenier $\mathrm{KH}$, et al. Copeptin, a surrogate marker for arginine vasopressin, is associated with cardiovascular and all-cause mortality in patients with type 2 diabetes (ZODIAC-31). Diabetes Care. 2013;36(10):3201-7.

118. Velho G, Bouby N, Hadjadj S, Matallah N, Mohammedi K, Fumeron F, et al. Plasma copeptin and renal outcomes in patients with type 2 diabetes and albuminuria. Diabetes Care. 2013;36(11):3639-45.

119. Plischke M, Kohl M, Bankir L, Shayganfar S, Handisurya A, Heinze G, et al. Urine osmolarity and risk of dialysis initiation in a chronic kidney disease cohort-a possible titration target? PLoS One. 2014;9(3):e93226.

120. Correa-Rotter R, Wesseling C, Johnson RJ. CKD of unknown origin in Central America: the case for a Mesoamerican nephropathy. Am J Kidney Dis. 2014;63(3):506-20.

121. Serradeil-Le Gal C, Wagnon J, Valette G, Garcia G, Pascal M, Maffrand JP, et al. Nonpeptide vasopressin receptor antagonists: development of selective and orally active V1a, V2 and V1b receptor ligands. Prog Brain Res. 2002;139:197210.

122. Verbalis JG. AVP receptor antagonists as aquaretics: review and assessment of clinical data. Cleve Clin J Med. 2006;73 Suppl 3:S24-33.

123. Aperis G, Alivanis P. Tolvaptan: a new therapeutic agent. Rev Recent Clin Trials. 2011;6(2):177-88.

124. Tsukaguchi H, Shayakul C, Berger UV, Hediger MA. Urea transporters in kidney: molecular analysis and contribution to the urinary concentrating process1. Am J Physiol. 1998;275(3 Pt 2):F319-24. 
Tableau 1. Caractéristiques différentes entre la pars recta et la partie contournée du tubule proximal

\section{Tubule contourné proximal (PCT) Pars recta (PST)}

\begin{tabular}{|c|c|c|}
\hline Situation & Cortex & $\begin{array}{l}\text { Rayons médullaires du cortex et zone externe de la médulla } \\
\text { externe }\end{array}$ \\
\hline Type d'épithelium & $\begin{array}{l}\text { S1 et S2 } \\
\text { Bordure en brosse bien développée }\end{array}$ & $\begin{array}{l}\text { S3 (+ S4 chez les carnivores) } \\
\text { Bordure en brosse plus réduite }\end{array}$ \\
\hline Environnement tubulaire & Tubules contournés distaux & $\begin{array}{l}\text { Segments larges ascendants de l'anse de Henle } \\
\text { Canaux collecteurs }\end{array}$ \\
\hline Oxygénation & Très bonne oxygénation & Oxygénation limitée \\
\hline Aquaporines & AQP1 basolatérale et apicale & $\begin{array}{l}\text { AQP1 basolatérale et apicale } \\
\text { AQP7 apicale (aquaglycéroporine) }\end{array}$ \\
\hline $\begin{array}{l}\text { Transporteur apical de } \\
\text { glucose }\end{array}$ & SGLT2 (transporte 1 glucose $+1 \mathrm{Na}$ ) & SGLT1 (transporte 1 glucose + $2 \mathrm{Na}$ ) \\
\hline
\end{tabular}




\begin{tabular}{lll}
\hline Fonctions de transport & $\begin{array}{l}\text { Réabsorption de divers solutés (ions, } \\
\text { glucose, acides aminés, albumine, } \\
\text { urée, etc.... }\end{array}$ & $\begin{array}{l}\text { Réabsorption de divers solutés (moindre que dans PCT) } \\
\text { Sécrétion d'acides organiques (exemple: hippurate), de } \\
\text { nucléotides, d'acide urique, de xénobiotiques, etc... } \\
\text { Secrétion très probable d'urée }\end{array}$ \\
& $\begin{array}{l}\text { L'eau suit de façon iso-osmotique } \\
\text { L'eau suit de façon iso-osmotique dans les deux directions. Si } \\
\text { les secrétions sont > aux réabsorptions, elles peuvent } \\
\text { augmenter le débit de fluide délivré au TDL }\end{array}$
\end{tabular}

Fonctions métaboliques Néoglucogénèse et ammoniogénèse possibles mais peu importantes en Néoglucogénèse et ammoniogénèse plus intenses que dans la conditions normales partie contournée

Production d'arginine à partir de

Formation de polyamines à partir d'ornithine (issue de citrulline luminale (cette arginine part l'hydrolyse d'arginine luminale, et non de l'arginine du PCT) dans le sang périphérique) 
Tableau 2. Caractéristiques des parties médullaire et corticale du segment large ascendant de l'anse de Henle

\section{TAL Médullaire dans la IS}

\begin{tabular}{ll}
\hline Situation & Zone interne de la médulla externe (IS) \\
& \\
\hline Type d'épithelium & Epithélium assez épais, extrèmement riche \\
& en mitochondries. \\
& Très grand développement de la membrane \\
& basolatérale avec nombreux replis \\
& Très peu de villosités sur la face apicale des \\
& cellules.
\end{tabular}

\section{Environnement tubulaire Segments grèles descendants dans la IS} Canaux collecteurs médullaires

\begin{tabular}{ll}
\hline Environnement & Réseau de capillaires issus des vasa recta \\
vasculaire & $\begin{array}{l}\text { descendants situés à la périphérie des } \\
\text { faisceaux vasculaires (l'oxygène n'est sans } \\
\text { doute que modestement shunté avec les } \\
\text { vasa recta ascendants). }\end{array}$
\end{tabular}

Osmolarité de

l'interstitium environnant

\section{TAL "Cortical" (incluant le TAL dans la OS)}

Partie située dans la OS puis rayons médullaires (RM) du cortex

Les néphrons profonds n'ont pratiquement pas de TAL cortical. Juste un peu dans la OS.

\section{Epithélium moins épais}

Mitochondries moins étroitement associées avec les repis de la membrane basolatérale.

Plus de villosités sur la membrane apicale des cellules

Pars recta du tubule proximal dans la OS et les RM Canaux collecteurs corticaux et de la OS

Capillaires corticaux issus des artérioles efférentes des glomérules superficiels et moyens. Bonne oxygénation. Sans doute moindre oxygénation dans la OS.

\begin{tabular}{lll}
\hline Propriétés de transport & $\begin{array}{l}\text { Capacité de transport de Na élevée } \\
\text { Conductance apicale au K élevée }\end{array}$ & $\begin{array}{l}\text { Capacité de transport de Na faible } \\
\text { Conductance apicale au K plus faible }\end{array}$
\end{tabular}




\begin{tabular}{lll}
\hline Isoformes de NKCC2 & F et A & B et A \\
\hline $\begin{array}{l}\text { Différence d'osmolarité } \\
\text { transépithéliale créée }\end{array}$ & Faible & Importante \\
\hline $\begin{array}{l}\text { Hormones peptidiques } \\
\text { (agissant sur AMPc) }\end{array}$ & $\begin{array}{l}\text { Glucagon, calcitonine, vasopressine } \\
\text { (pas de réponse à la PTH) }\end{array}$ & Glucagon, calcitonine, vasopressine, PTH \\
\hline $\begin{array}{l}\text { Adaptation fonctionnelle } \\
\text { en réponse à une } \\
\begin{array}{l}\text { augmentation de l'activité } \\
\text { de concentration urinaire }\end{array}\end{array}$ & $\begin{array}{l}\text { Hypertrophie des cellules et augmentation du } \\
\text { nombre de cellules par section de MTAL. } \\
\text { binuclées }\end{array}$ & $\begin{array}{l}\text { Pas d'hypertrophie, et même relative atrophie dans la nombre de cellules } \\
\text { partie la plus distale du CTAL }\end{array}$ \\
\hline
\end{tabular}

\section{Pas de différence connue entre les deux sous-segments pour les fonctions suivantes}

\begin{tabular}{ll}
\hline Permeabilité à l'eau & Impermeabilité à l'eau \\
\hline Fonction de transport & Réabsorption active de $\mathrm{NaCl}$, entraînant une réabsorption secondaire de Ca et Mg \\
\hline Fonctions métaboliques & Synthèse de l'uromoduline (protéine de Tamm-Horsfall) et sécrétion dans la lumière
\end{tabular}




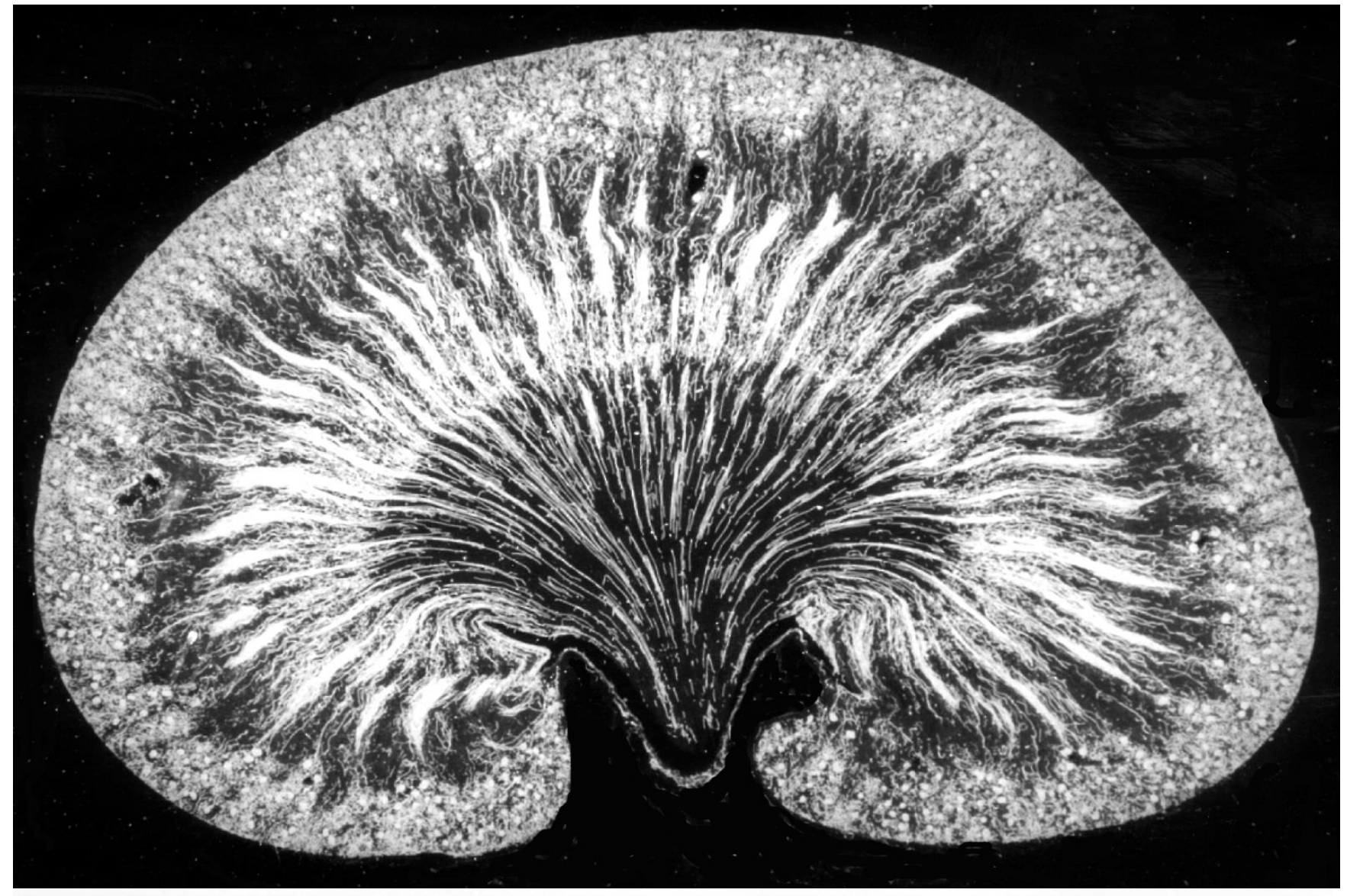




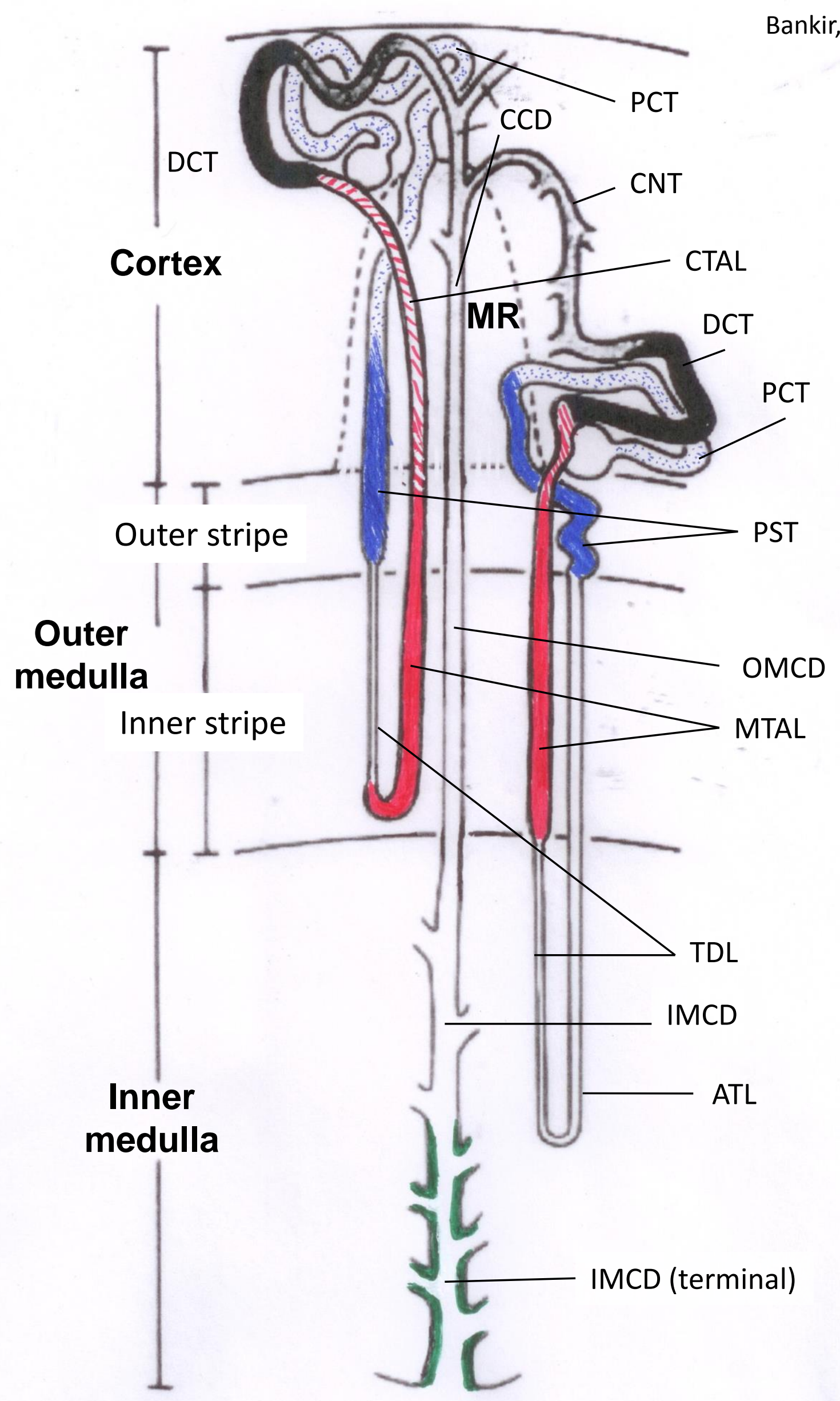



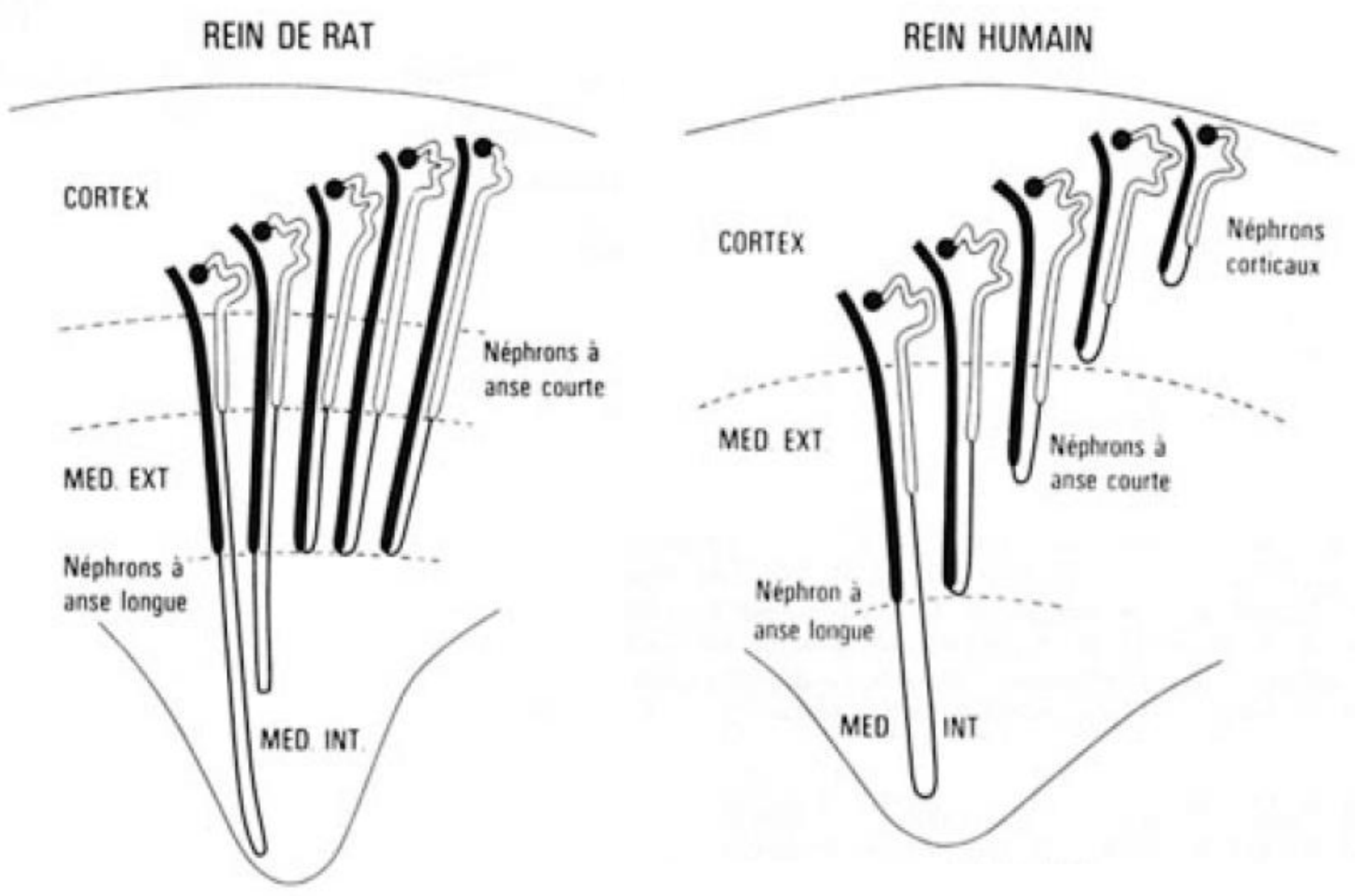


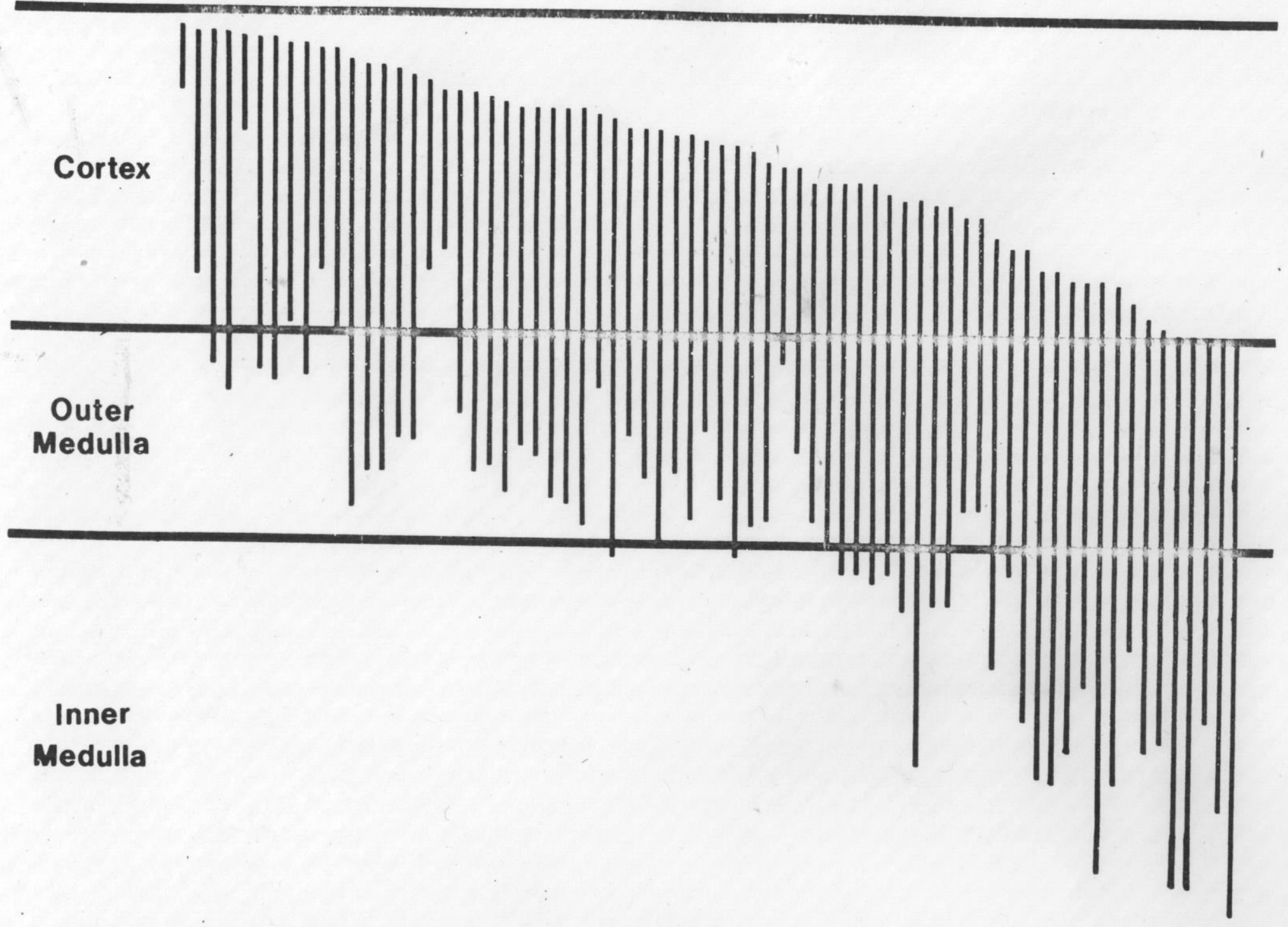


Bankir, Figure 5

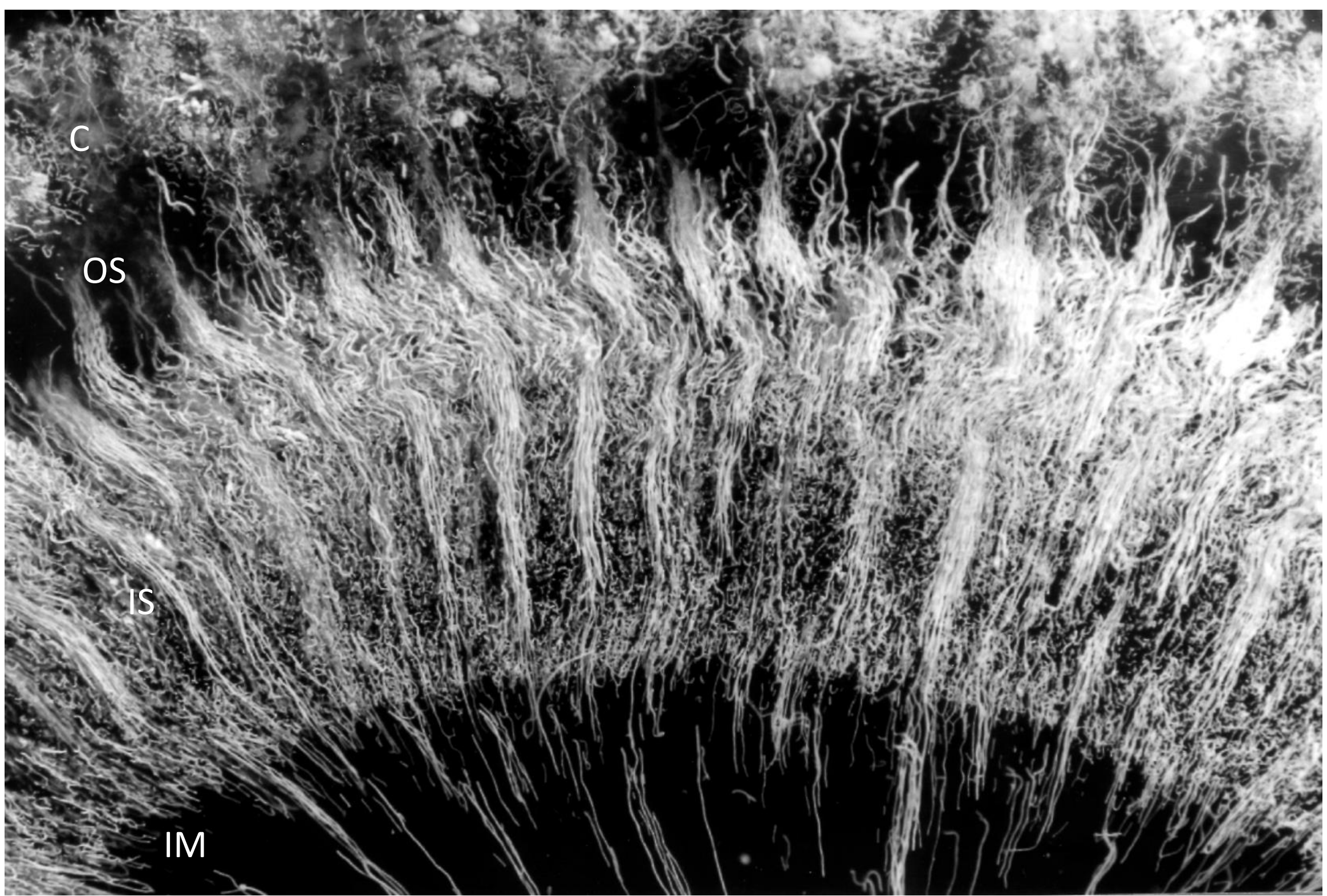



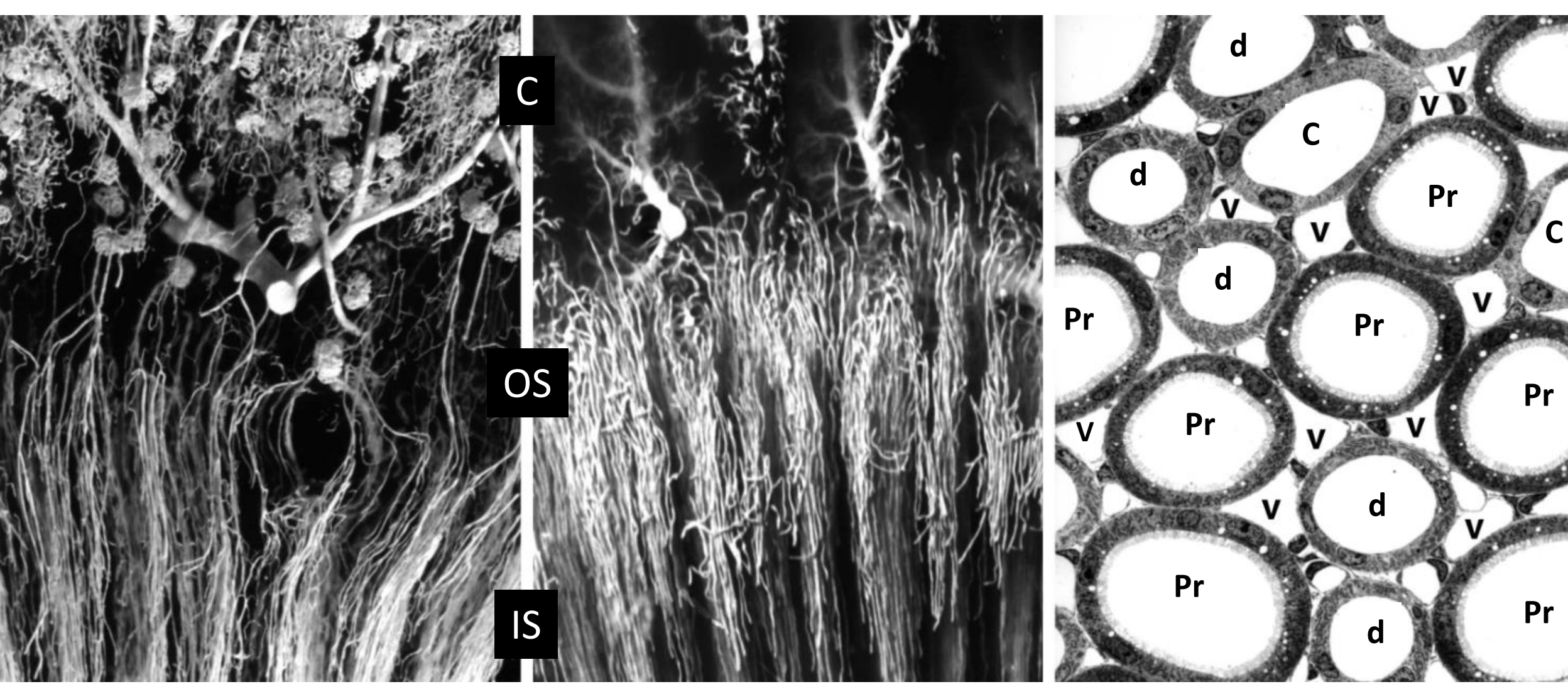
Bankir, Figure 7
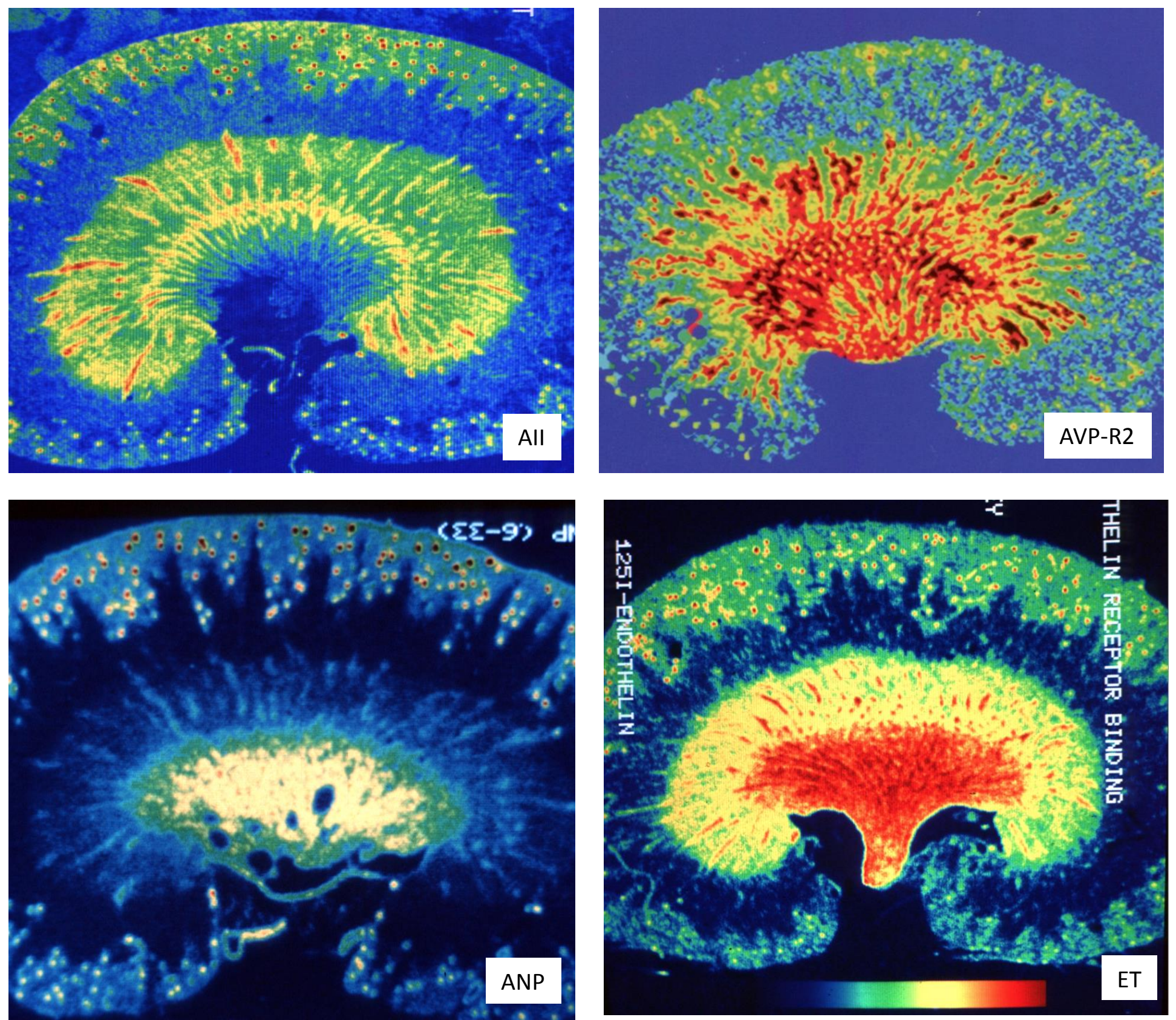


\section{AMPHIBIENS}
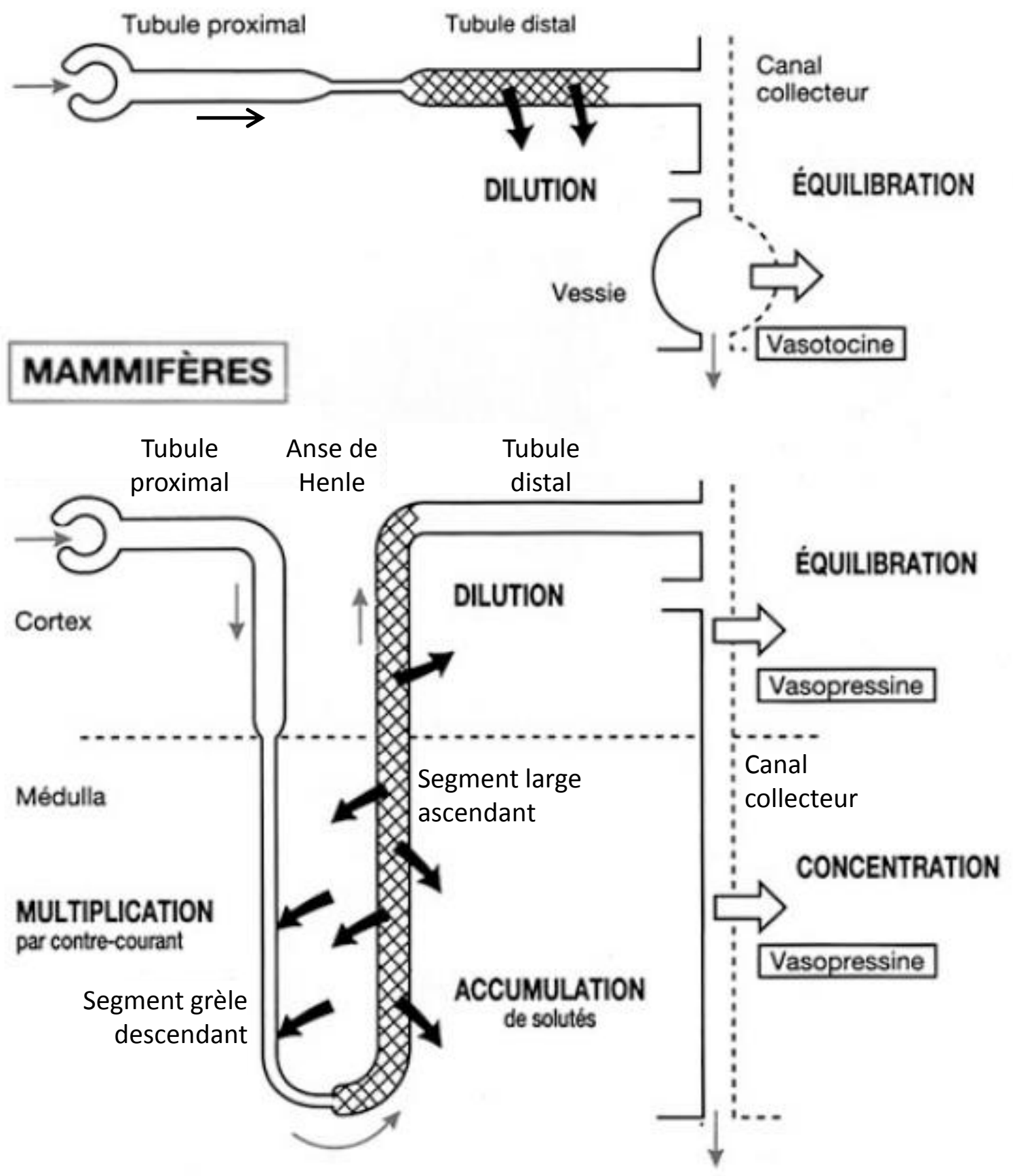


\section{UT-A1/3}

Bankir, Figure 9

\section{UT-A2}
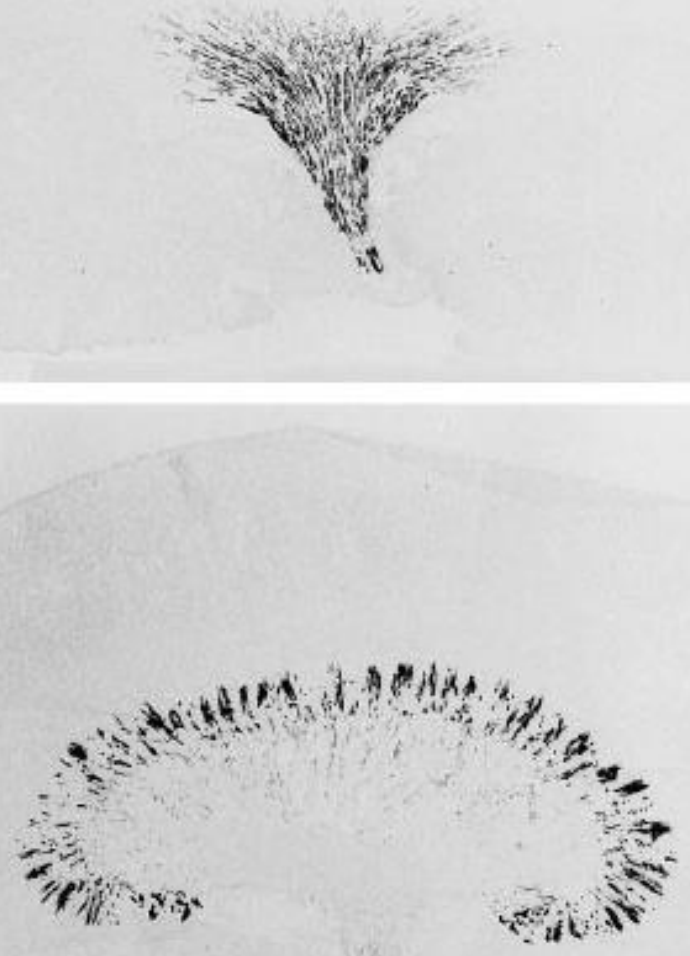

UT-B

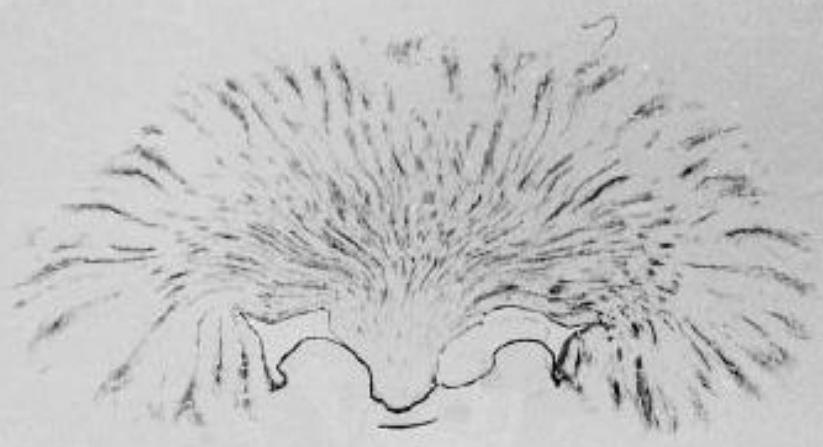


Bankir, Figure 10

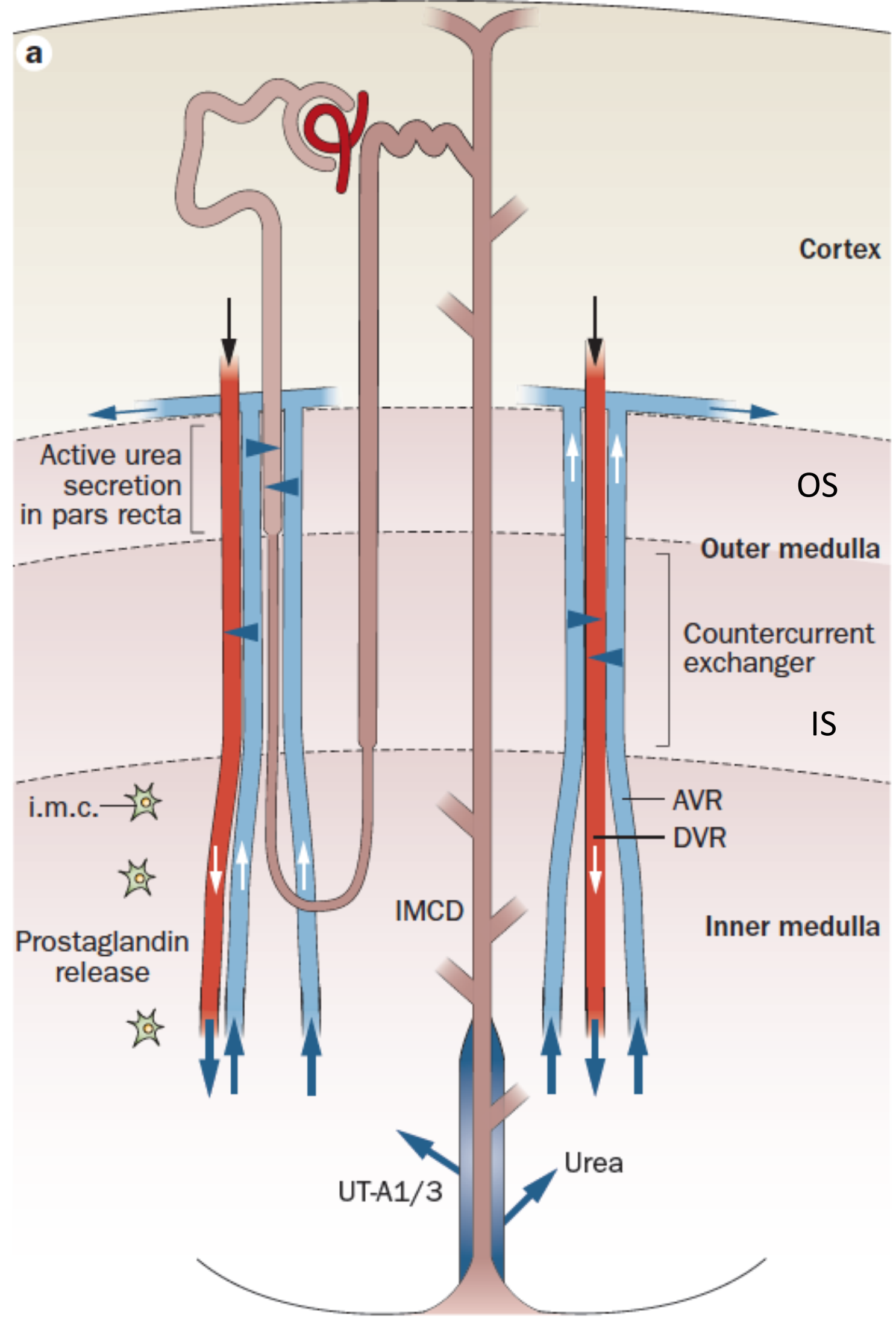

